Draft Version November 8, 2017

Preprint typeset using $\mathrm{LAT}_{\mathrm{E}} \mathrm{X}$ style emulateapj v. 5/2/11

\title{
LINEAR STABILITY OF MAGNETIZED MASSIVE PROTOPLANETARY DISKS
}

\author{
MiN-KAI LiN \\ Canadian Institute for Theoretical Astrophysics, 60 St. George Street, Toronto, ON, M5S 3H8, Canada \\ Draft version November 8, 2017
}

\begin{abstract}
Magneto-rotational instability (MRI) and gravitational instability (GI) are the two principle routes to turbulent angular momentum transport in accretion disks. Protoplanetary disks may develop both. This paper aims to reinvigorate interest in the study of magnetized massive protoplanetary disks, starting from the basic issue of stability. The local linear stability of a self-gravitating, uniformly magnetized, differentially rotating, three-dimensional stratified disk subject to axisymmetric perturbations is calculated numerically. The formulation includes resistivity. It is found that the reduction in the disk thickness by self-gravity can decrease MRI growth rates; the MRI becomes global in the vertical direction, and MRI modes with small radial length scales are stabilized. The maximum vertical field strength that permits the MRI in a strongly self-gravitating polytropic disk with polytropic index $\Gamma=1$ is estimated to be $B_{z, \max } \simeq c_{s 0} \Omega \sqrt{\mu_{0} / 16 \pi G}$, where $c_{s 0}$ is the midplane sound speed and $\Omega$ is the angular velocity. In massive disks with layered resistivity, the MRI is not well-localized to regions where the Elsasser number exceeds unity. For MRI modes with radial length scales on the order of the disk thickness, self-gravity can enhance density perturbations, an effect that becomes significant in the presence of a strong toroidal field, and which depends on the symmetry of the underlying MRI mode. In gravitationally unstable disks where GI and MRI growth rates are comparable, the character of unstable modes can transition smoothly between MRI and GI. Implications for non-linear simulations are discussed briefly.
\end{abstract}

\section{INTRODUCTION}

Astrophysical disks host a wide range of fluid instabilities. Among them, the magneto-rotational instability (MRI, Chandrasekhar 1961; Balbus \& Hawlev 1991, 1998) and gravitational instability (GI, Toomre 1964; Goldreich \& Lynden-Bell 1965a, b) provide robust pathways to turbulent angular momentum transport that enables mass accretion (Balbus \& Papaloizou 1999; Armitage 2011; Turner et al. 2014, and references therein). They are also relevant to planet formation theory. For example, the strength of MRI turbulence directly affect planetesimal dynamics in protoplanetary disks (Yang et al. 2012; Gressel et al. 2012); while GI can potentially form giant planets directly through disk fragmentation (Boss 1997, 1998; Gammie 2001; Vorobyov 2013; Helled et al. 2013).

Accretion disks such as those surrounding black holes can develop both MRI and GI (Menou \& Quataert 2001; Goodman 2003). Protoplanetary disks (PPDs) are also expected to be massive and magnetized in its earliest evolutionary phase (Inutsuka et al. 2010). The interplay between MRI and GI has been invoked to explain outbursts in circumstellar disks (Armitage et al. 2001; Zhu et al. 2010b.a; Martin et al. 2012b), and predicts similar phenomenon in circumplanetary disks (Lubow \& Martin 2012). This results from the development of 'dead zones' - magnetically inactive, laminar regions near the disk midplane - with magnetized layers above and below (Gammie 1996a; Martin et al. 2012a; Landry et al. 2013). Mass accumulation in the dead zone can lead to GI and trigger MRI through heating. In these models, the condition required for MRI is realized through GI, but the MRI is unaffected by disk self-gravity.

mklin924@cita.utoronto.ca
PPDs subject to both MRI and GI are often modeled through separate turbulent viscosity coefficients in a hydrodynamical framework (Terquem 2008). This implicitly assumes that the development of MRI and GI can be assessed independently. Circumstellar disk models that explicitly combine the equations of magnetohydrodynamics and self-gravity have been limited to a few early simulations (Fromang et al. 2004c, a; Fromang 2005). It will be necessary to revisit and extend these pioneering calculations to fully explore the impact of MRI and GI on the structure and evolution of PPDs. In preparation of this, it is important to have a thorough understanding of the stability properties of such systems.

Since compressibility is not fundamental for the MRI, much of the early stability calculations assume incompressible perturbations (Goodman \& Xu 1994; Jin 1996). However, recent works indicate compressibility may be important under certain conditions, such as strong fields (Kim \& Ostriker 2000; Pessah \& Psaltis 2005; Bonanno \& Urpin 2007). Previous MRI studies have also focused on modes with vanishing radial wavenumber, because they are the most unstable (Sano \& Miyama 1999; Reyes-Ruiz 2001). Self-gravity has minimal effect on such perturbations in a rotating disk. However, modes with radial length scales on the order of the disk scale height may be subject to selfgravity. It is therefore of interest to generalize the MRI with non-zero radial wavenumbers to massive disks.

The effect of a magnetic field on the GI of rotating disks has been considered recently by Lizano et al. (2010), who generalized the Toomre stability criterion for razor-thin disks to include a vertical field. For circumstellar disks, the authors concluded that the field is stabilizing. This is consistent with previous analysis by Nakamura (1983) for three-dimensional (3D) uniformly rotating disks. How- 
ever, the GI of 3D differentially rotating disks have mostly neglected magnetic fields (Mamatsashvili \& Rice 2010; Kim et al. 2012), but such disks are subject to the MRI if magnetized.

This work marks the beginning of our study of magnetized, self-gravitating PPDs. We start from linear calculations, which have the advantage that a wide range of parameters can be studied at negligible computational cost. This allows us to identify conditions, if any, under which MRI and GI cannot be considered independent. It is also important to have such calculations to benchmark and guide future non-linear simulations.

This paper is organized as follows. \$2 lists the governing equations and describes the disk equilibria under consideration. The linear problem is formulated in $\$ 3$. The impact of self-gravity on the MRI with a vertical field is discussed in $\$ 4$, gravitationally unstable disks are considered in \$5, and equilibria including an azimuthal field is explored in 8 . We summarize results in $\$ 7$ with a discussion of important extensions to our current models.

\section{LOCAL DISK MODEL}

We study the local stability of an inviscid, selfgravitating and magnetized fluid disk orbiting a central star with potential $\Phi_{*}(r, z)$, where $(r, \varphi, z)$ are cylindrical co-ordinates from the star. We use the shearing box approximation (Goldreich \& Lynden-Bell 1965b) to consider a small patch of the disk at a fiducial radius $r=r_{0}$. The local frame rotates at angular velocity $\Omega_{0}=\Omega\left(r_{0}, 0\right)$ about the star, where $r \Omega^{2}=\partial \Phi_{*} / \partial r$. We also define $S \equiv-r \partial \Omega / \partial r$ as the local shear rate and $\Omega_{z}^{2} \equiv \partial^{2} \Phi_{*} / \partial z^{2}$ as the square of the local vertical frequency.

A Cartesian co-ordinate system $(x, y, z)$ is set up in this local frame, corresponding to the radial, azimuthal and vertical directions of the global disk, respectively. The shearing box fluid equations read

$$
\begin{aligned}
& \frac{\partial \rho}{\partial t}+\nabla \cdot(\rho \boldsymbol{v})=0 \\
& \frac{\partial \boldsymbol{v}}{\partial t}+\boldsymbol{v} \cdot \nabla \boldsymbol{v}+2 \Omega_{0} \hat{\boldsymbol{z}} \times \boldsymbol{v}=-\frac{1}{\rho} \nabla \Pi+\frac{1}{\rho \mu_{0}} \boldsymbol{B} \cdot \nabla \boldsymbol{B}-\nabla \Phi
\end{aligned}
$$$$
\frac{\partial \boldsymbol{B}}{\partial t}=\nabla \times(\boldsymbol{v} \times \boldsymbol{B}-\eta \nabla \times \boldsymbol{B}),
$$

where $\rho$ is the density field; $\boldsymbol{v}$ is the total velocity in the local frame; $\boldsymbol{B}$ is the magnetic field which satisfies $\nabla \cdot \boldsymbol{B}=0 ; \Pi \equiv P+|\boldsymbol{B}|^{2} / 2 \mu_{0}$ is the total pressure, and $\mu_{0}$ is the vacuum permeability. We choose a barotropic equation of state, specified below, so that the gas pressure is given by $P=P(\rho)$. The resistivity $\eta$ is either uniform or a prescribed function of height.

The total potential is $\Phi=\Phi_{\text {ext }}+\Phi_{d}$, where

$$
\Phi_{\text {ext }}(x, z)=-\Omega_{0} S_{0} x^{2}+\frac{1}{2} \Omega_{z 0}^{2} z^{2}
$$

is the effective external potential (central plus centrifugal) in the shearing box approximation, where $S_{0} \equiv S\left(r_{0}, 0\right)$ and $\Omega_{z 0} \equiv \Omega_{z}\left(r_{0}, 0\right)$; and the gas potential $\Phi_{d}$ satisfies Poisson's equation

$$
\nabla^{2} \Phi_{d}=4 \pi G \rho
$$

where $G$ is the gravitational constant. For clarity, hereafter we drop the subscript 0 on the frequencies.

\subsection{Equilibrium disk}

The unperturbed disk is steady and described by $\rho=\rho(z), \boldsymbol{B}=B_{z} \hat{\boldsymbol{z}}+B_{y} \hat{\boldsymbol{y}}$ where $B_{y, z}$ are constants and the toroidal field strength is $B_{y}=\epsilon B_{z}$. The equilibrium velocity field is $\boldsymbol{v}=-S x \hat{\boldsymbol{y}}$. We consider Keplerian disks so that $S=3 \Omega / 2$ and the epicycle frequency $\kappa \equiv \sqrt{2 \Omega(2 \Omega-S)}=\Omega=\Omega_{z}$. We assume a thin disk and neglect the radial component of the self-gravitational force in the unperturbed disk.

The equilibrium density field is obtained by solving

$$
\begin{aligned}
& 0=\frac{1}{\rho} \frac{d P}{d z}+\Omega_{z}^{2} z+\frac{d \Phi_{d}}{d z}, \\
& \frac{d^{2} \Phi_{d}}{d z^{2}}=4 \pi G \rho .
\end{aligned}
$$

We consider (i) isothermal disks with $P=c_{s 0}^{2} \rho$; (ii) polytropic disks with $P=K \rho^{2}$ with $K=c_{s 0}^{2} / 2 \rho_{0}$; where $\rho_{0} \equiv \rho(0)$ is the midplane density. The sound speed $c_{s} \equiv \sqrt{d P / d \rho}$ so that $c_{s 0}$ is the global sound speed in the isothermal disk, and is the midplane sound speed in the polytropic disk. For the polytropic disk the disk thickness $H$ is such that $\rho(H)=0$. Since the isothermal disk has no surface, we define $H$ such that $\rho(H)=10^{-2} \rho_{0}$. A non-dimensional measure of the disk thickness is given by

$$
f^{-1}=\frac{H \Omega}{c_{s 0}},
$$

and $f$ will appear in subsequent discussions.

We solve for $\hat{\rho} \equiv \rho / \rho_{0}$ with boundary conditions $\hat{\rho}=$ 1 and $d \hat{\rho} / d z=0$ at $z=0$. This is done numerically for isothermal disks and analytically for the polytropic disk (see Appendix A). Examples of density profiles are shown in Fig. 1. The normalized density field is weakly dependent on the strength of self-gravity provided the $z$-axis is appropriately scaled.

\subsection{Resistivity profile}

We adopt constant resistivity or a resistivity prescription such that $\eta(z)$ increase towards the midplane. In the latter case, we follow Fleming \& Stone (2003) and use the resistivity profile

$$
\eta(z)=\sqrt{2} \eta_{0}\left[\exp \left(-g_{+}\right)+\exp \left(-g_{-}\right)\right]^{-1 / 2},
$$

where

$$
\begin{aligned}
& g_{ \pm}(z)=\frac{\Sigma_{ \pm}(z)-\Sigma_{0}}{\Sigma_{*}} \\
& \Sigma_{ \pm}(z)=\int_{ \pm z}^{\infty} \rho\left(z^{\prime}\right) d z^{\prime}
\end{aligned}
$$

and $\Sigma_{0} \equiv \Sigma_{ \pm}(0)$, so that $g_{ \pm}(0)=0$ and $\eta_{0}=\eta(0)$. The constant $\Sigma_{*}$ is chosen such that

$$
\cosh \left(\frac{\Sigma_{0}}{\Sigma_{*}}\right)=\left[\frac{\eta_{0}}{\eta(\infty)}\right]^{2},
$$

and we define $\eta_{0} / \eta(\infty) \equiv A$ as the conductivity boost factor from the midplane to the disk surface. We remark that once $\rho$ and $d \rho / d z$ are obtained from Eq. 6 - 7 . the integration for Eq. 11 can be performed implicitly by using Poisson's equation. 


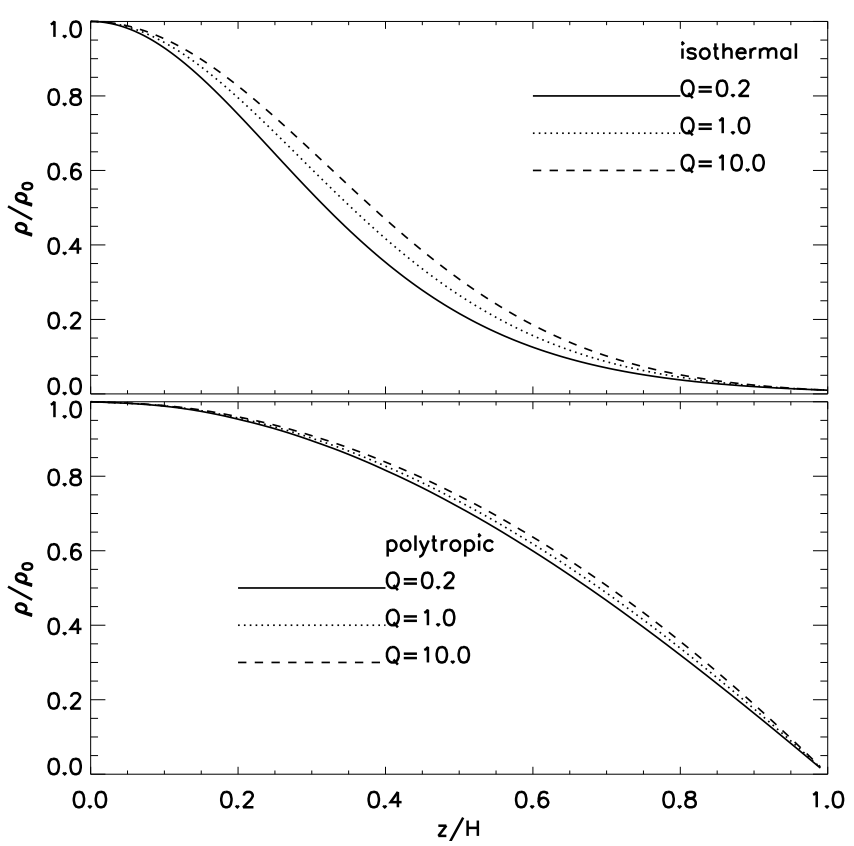

Figure 1. Equilibrium density field from solving Eq. 6- 7 subject to an isothermal (top) and polytropic (bottom) equation of state. Note that the normalization for the horizontal axis also depends on the strength of self-gravity, i.e. $H=H(Q)$ and is an increasing function of $Q$.

We use the Elsasser number $\Lambda$ a non-dimensional measure of conductivity,

$$
\Lambda \equiv \frac{v_{A}^{2}}{\eta \Omega}
$$

where $v_{A} \equiv B_{z} / \sqrt{\mu_{0} \rho}$ is the vertical Alfven speed. Because of the density stratification, the Elsasser number increases with height even for constant resistivity. The disk may be considered ideal where $\Lambda \gtrsim 1$.

\subsection{Disk parameters}

The strength of self-gravity is parametrized by

$$
Q \equiv \frac{\Omega^{2}}{4 \pi G \rho_{0}}
$$

(Mamatsashvili \& Rice 2010), which is used to set the midplane density $\rho_{0}$. A relation between $Q$ and the Toomre parameter for gravitational instability of razorthin disks, $Q_{2 \mathrm{D}}$, is described in Appendix $\mathrm{B}$.

The plasma $\beta$ measures the inverse strength of the magnetic field

$$
\beta \equiv \frac{c_{s 0}^{2}}{v_{A 0}^{2}}=\frac{c_{s 0}^{2} \mu_{0} \rho_{0}}{B_{z}^{2}}
$$

where $v_{A 0}$ is the midplane Alfven speed. Note that we use the vertical field for this definition throughout this paper.

The strength of conductivity is measured by the midplane Elsasser number

$$
\Lambda_{0} \equiv \Lambda(0)=\frac{v_{A 0}^{2}}{\eta_{0} \Omega} .
$$

For non-uniform resistivity we also specify $A>1$.

\section{LINEAR PROBLEM}

We consider axisymmetric Eulerian perturbations to the above equilibrium in the form $\operatorname{Re}\left[\delta \rho(z) \exp \mathrm{i}\left(k_{x} x+\sigma t\right)\right]$ and similarly for other fluid variables. Here, $k_{x}$ is a constant radial wavenumber and $\sigma=-(\omega+\mathrm{i} \gamma)$ is a complex frequency, where $-\omega$ is the real mode frequency and $\gamma$ is the growth rate. We take $k_{x}>0$ without loss of generality. Hereafter, we suppress the exponential factor and the real part notation.

The linearized continuity equation is

$$
\frac{\mathrm{i} \sigma}{c_{s}^{2}} W+\mathrm{i} k_{x} \delta v_{x}+(\ln \rho)^{\prime} \delta v_{z}+\delta v_{z}^{\prime}=0
$$

where ' denotes $d / d z$ and $W=\delta P / \rho=c_{s}^{2} \delta \rho / \rho$ is the enthalpy perturbation. The linearized equations of motion are

$\mathrm{i} \sigma \delta v_{x}-2 \Omega \delta v_{y}=-\mathrm{i} k_{x} \widetilde{W}+\frac{B_{z}}{\mu_{0} \rho}\left[\delta B_{x}^{\prime}-\mathrm{i} k_{x}\left(\delta B_{z}+\epsilon \delta B_{y}\right)\right]$,

$\mathrm{i} \sigma \delta v_{y}+\frac{\kappa^{2}}{2 \Omega} \delta v_{x}=\frac{B_{z}}{\mu_{0} \rho} \delta B_{y}^{\prime}$

$\mathrm{i} \sigma \delta v_{z}=-\widetilde{W^{\prime}}-\frac{B_{y}}{\mu_{0} \rho} \delta B_{y}^{\prime}$

where the effective enthalpy perturbation $\widetilde{W}=W+\delta \Phi$. The components of the linearized induction equation are

$$
\begin{aligned}
& \mathrm{i} \bar{\sigma} \delta B_{x}=B_{z} \delta v_{x}^{\prime}+\eta \delta B_{x}^{\prime \prime}+\eta^{\prime} \delta B_{x}^{\prime}-\mathrm{i} k_{x} \eta^{\prime} \delta B_{z}, \\
& \mathrm{i} \bar{\sigma} \delta B_{y}=B_{z} \delta v_{y}^{\prime}-B_{y} \Delta-S \delta B_{x}+\eta \delta B_{y}^{\prime \prime}+\eta^{\prime} \delta B_{y}^{\prime}, \\
& \mathrm{i} \bar{\sigma} \delta B_{z}=-\mathrm{i} k_{x} B_{z} \delta v_{x}+\eta \delta B_{z}^{\prime \prime},
\end{aligned}
$$

where $\mathrm{i} \bar{\sigma}=\mathrm{i} \sigma+\eta k_{x}^{2}, \Delta \equiv \nabla \cdot \boldsymbol{\delta} \boldsymbol{v}=\mathrm{i} k_{x} \delta v_{x}+\delta v_{z}^{\prime}$, and the divergence-free condition is $\mathrm{i} k_{x} \delta B_{x}+\delta B_{z}^{\prime}=0$. Finally, the linearized Poisson equation is

$$
\delta \Phi^{\prime \prime}-k_{x}^{2} \delta \Phi=\frac{\Omega^{2} \rho}{c_{s}^{2} Q \rho_{0}} W .
$$

We eliminate $\delta \boldsymbol{B}$ and $\delta v_{z}$ between the linearized equations to obtain a system of ordinary differential equations for $\boldsymbol{U}=\left(\delta v_{x}, \delta v_{y}, W, \delta \Phi\right)$. We detail the steps in Appendix $\mathrm{C}$ for two cases considered in this paper:

1. Purely vertical field with constant or variable resistivity, so that $\epsilon=0$ and $\eta=\eta(z)$.

2. Tilted field with uniform resistivity so that $\epsilon \neq 0$ and $\eta=$ constant.

Schematically, the numerical problem is to solve

$$
\begin{aligned}
L_{11} \delta v_{x}+L_{12} \delta v_{y}+L_{13} W+L_{14} \delta \Phi & =0, \\
L_{21} \delta v_{x}+L_{22} \delta v_{y}+L_{23} W+L_{24} \delta \Phi & =0, \\
L_{31} \delta v_{x}+L_{32} \delta v_{y}+L_{33} W+L_{34} \delta \Phi & =0, \\
L_{43} W+L_{44} \delta \Phi & =0,
\end{aligned}
$$

where the differential operators $L_{1 j}, L_{2 j}$ and $L_{3 j}(j=$ $1,2,3,4)$ can be read off Appendix C and $L_{4 j}(j=3,4)$ from the linearized Poisson equation above. We remark that the case of a tilted field and variable resistivity can also be reduced to the above form. 


\subsection{Domain and boundary conditions}

For a vertical field, considered in $\$ 4$ and $\$ 5$, we take $\boldsymbol{U}$ to be an even function of $z$. Odd modes are permitted in 96. where an azimuthal field may be included. In both setups the gravitational potential boundary condition, given by Goldreich \& Lynden-Bell (1965a), is

$$
\delta \Phi^{\prime}\left( \pm Z_{s}\right) \pm k_{x} \delta \Phi\left( \pm Z_{s}\right)=-\left.\frac{\Omega^{2} \rho \xi_{z}}{\rho_{0} Q}\right|_{ \pm Z_{s}}
$$

where $\xi_{z}=\delta v_{z} / \mathrm{i} \sigma$ is the vertical Lagrangian displacement, and $z= \pm Z_{s}$ is the upper and lower disk surfaces, respectively.

\subsubsection{Case 1: vertical field}

Here we impose $d \boldsymbol{U} / d z=0$ at $z=0$. This permits higher numerical resolution by reducing the computational domain to $z \in\left[0, Z_{s}\right]$. At the upper disk boundary $z=Z_{s}$ we set

$$
\delta B_{x}\left(Z_{s}\right)=\delta B_{y}\left(Z_{s}\right)=\delta v_{z}\left(Z_{s}\right)=0,
$$

so the field remains vertical. The derivation of the magnetic field boundary conditions may be found in Sano \& Mivama (1999).

\subsubsection{Case 2: tilted field}

In this more general setup the computational domain is $z \in\left[-Z_{s}, Z_{s}\right]$ and no symmetry across the midplane is enforced. At the disk surfaces we adopt the 'halo' model of Gammie \& Balbus (1994), so that

$$
\begin{aligned}
\Delta\left( \pm Z_{s}\right) & =0, \\
\delta B_{y}\left( \pm Z_{s}\right) & =0, \\
\delta B_{z}\left( \pm Z_{s}\right) \mp \mathrm{i} \delta B_{x}\left( \pm Z_{s}\right) & =0,
\end{aligned}
$$

and this case permits $\delta v_{z}\left( \pm Z_{s}\right) \neq 0$

\subsection{Numerical procedure}

We use a pseudo-spectral method to solve the set of linearized equations. Let

$$
\boldsymbol{U}(z)=\sum_{k=1}^{N_{z}} \boldsymbol{U}_{k} \psi_{k}\left(z / Z_{s}\right),
$$

where

$$
\psi_{k}= \begin{cases}T_{2(k-1)} & B_{y} \equiv 0(\text { case } 1), \\ T_{k-1} & B_{y} \neq 0(\text { case } 2),\end{cases}
$$

and $T_{l}$ is a Chebyshev polynomial of the first kind of order $l$ (Abramowitz \& Stegun 1965). Note that for case 1 the midplane symmetry condition is taken care of by the choice of basis functions.

The pseudo-spectral coefficients $\boldsymbol{U}_{n}$ are obtained by demanding the set of linear equations to be satisfied at $N_{z}$ collocation points along the vertical direction, here chosen to be the extrema of $T_{l_{\max }}$ plus end points, where $l_{\max }$ is the highest polynomial order. Our standard resolution is $N_{z}=256\left(N_{z}=257\right)$ for case 1 (case 2$)$.

The above procedure discretize the linear equations to a matrix equation,

$$
M w=0,
$$

where $M$ is a $4 N_{z} \times 4 N_{z}$ matrix representing the $L_{i j}$ plus boundary conditions, and $\boldsymbol{w}$ is a vector storing the pseudo-spectral coefficients. Starting with an initial guess for $\sigma$, non-trivial solutions to Eq. 36 are obtained by varying $\sigma$ using Newton-Raphson iteration such that $\operatorname{det} M=0$ (details can be found in Lin 2012).

\subsubsection{Non-dimensionalization}

We solve the linearized equations in non-dimensional form, by defining

$$
\begin{aligned}
& z=\hat{z} H, \quad k_{x}=\hat{k}_{x} / H, \quad \sigma=\hat{\sigma} \Omega, \quad \delta \boldsymbol{v}=c_{s 0} \delta \hat{\boldsymbol{v}} \\
& \delta \boldsymbol{B}=B_{z} \delta \hat{\boldsymbol{B}}, \quad \delta \rho=\rho \hat{W} / \hat{c}_{s}^{2}, \quad \delta \Phi=c_{s 0}^{2} \delta \hat{\Phi}
\end{aligned}
$$

where $\hat{c}_{s}=c_{s} / c_{s 0}$. We also non-dimensionalize background quantities, i.e. $\hat{v}_{A}=v_{A} / c_{s 0}, \hat{S}=S / \Omega, \hat{\kappa}=\kappa / \Omega$, $\hat{\Omega}_{z}=\Omega_{z} / \Omega$ and $\hat{\eta}=\eta /\left(H^{2} \Omega\right)$.

\subsection{Diagnostics}

We visualize results in terms of dimensionless energy densities. We define

$$
\begin{aligned}
& E_{m} \equiv \frac{|\delta \hat{\boldsymbol{B}}|^{2}}{2 \beta}, \\
& E_{g}=\frac{\hat{\rho}}{2 \hat{c}_{s}^{2}}\left|\operatorname{Re}\left(\hat{W} \delta \hat{\Phi}^{*}\right)\right|, \\
& E_{k}=\frac{1}{2} \hat{\rho}|\delta \hat{\boldsymbol{v}}|^{2}, \\
& E_{t}=\frac{\hat{\rho}|\hat{W}|^{2}}{2 \hat{c}_{s}^{2}},
\end{aligned}
$$

as the perturbed magnetic, gravitational, kinetic and thermal energies, respectively, which are functions of $z$. Although we do not solve an energy equation, we nevertheless define $E_{t}$ as a measure of density perturbations (Kojima et al. 1989). The total energy is $E=$ $E_{m}+E_{g}+E_{k}+E_{t}$. We use $\langle\cdot\rangle$ to denote an average over $z$.

Since we will primarily be concerned with massive disks, we define

$$
\tau \equiv \frac{\left\langle E_{g}\right\rangle}{\left\langle E_{g}\right\rangle+\left\langle E_{m}\right\rangle}
$$

as a measure of the importance of self-gravity. Thus, modes with $\tau=1$ are energetically dominated by selfgravity (GI) and modes with $\tau \ll 1$ are dominated by magnetic perturbations (MRI).

\section{MRI IN SELF-GRAVITATING DISKS}

In this section we focus on the MRI and use the vertical field setup of case 1 . We first consider MRI modes with negligible density/potential perturbations to see the effect of self-gravity on the MRI through the background stratification, then go on to examine MRI modes with density/potential perturbations in massive disks.

\subsection{Influence of self-gravity on the MRI through the background equilibrium}

Here we use polytropic disks, which have a well-defined disk thickness. The upper disk boundary is set to $Z_{s}=$ $0.99 H$. We fix $\beta=100$ and $k_{x} H=0.1$ unless otherwise stated. 


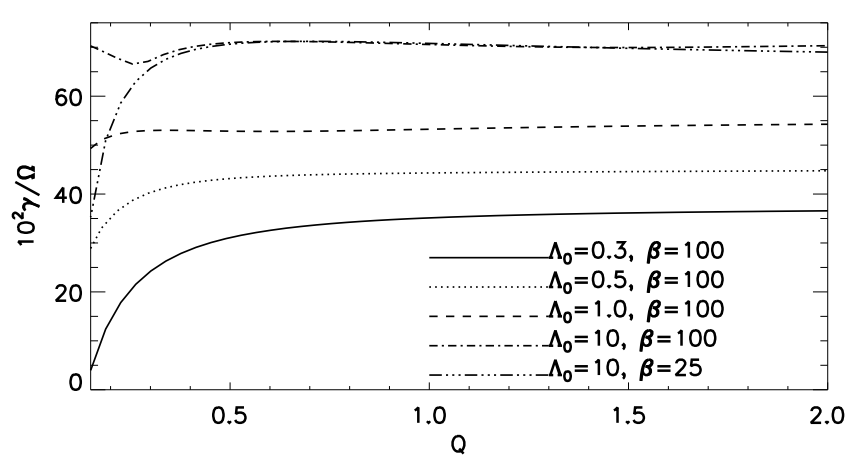

Figure 2. MRI growth rates as a function of $Q$ and midplane Elsasser numbers $\Lambda_{0}$, in polytropic disks with $\beta=100$ (solid, dotted and dot-dashed lines). The dash-triple dot line is the $\Lambda_{0}=10$ case with $\beta=25$. The resistivity is uniform.

\subsubsection{Uniform resistivity}

Fig. 2 plots MRI growth rates as a function of $Q$ and $\Lambda_{0}$. The resistivity is uniform $(A=1)$. For ideal MHD and a weak field $\left(\Lambda_{0}>1, \beta=100\right)$, there is negligible dependence on $Q$. However, with $\beta=25$ or in the resistive limit $\left(\Lambda_{0}<1\right)$, growth rates decrease noticeably for $Q<0.5\left(Q_{2 \mathrm{D}} \lesssim 1.5\right)$. Since we find density and potential perturbations to be negligible (i.e. the linear response is non-self-gravitating), this shows that disk self-gravity can affect the MRI through the background equilibrium.

Sano \& Miyama (1999) found that for MRI to operate, its wavelength $\lambda$ should fit inside the disk. That is,

$$
\lambda \equiv \max \left(\lambda_{\text {ideal }}, \lambda_{\text {resis }}\right) \lesssim 2 H,
$$

where the MRI wavelengths are given by

$$
\frac{\lambda_{\text {ideal }}}{2 H}=\frac{4 \pi}{\sqrt{15}} f \hat{v}_{A}=\frac{4 \pi f}{\sqrt{15 \beta \hat{\rho}}}
$$

for ideal MHD, and

$$
\frac{\lambda_{\text {resis }}}{2 H}=\frac{2 \pi}{\sqrt{3}} \frac{\hat{\eta}}{\hat{v}_{A} f}=\frac{2 \pi f}{\Lambda_{0}} \sqrt{\frac{\hat{\rho}}{3 \beta}}
$$

in the limit of high resistivity.

Because $\hat{\rho}$ is weakly dependent on $Q$ (Fig. 1), selfgravity only affects the MRI through the factor $f$, which increases with decreasing $Q$ (see Fig. 17 in Appendix A). This implies that sufficiently strong self-gravity can stabilize the MRI by making $2 H<\lambda$.

In the ideal limit with $\beta=100$, we find $\lambda<2 H$ throughout most of the disk for the values of $Q$ considered, so self-gravity does not affect growth rates significantly. However, the ratio $\lambda / 2 H$ does increase with stronger self-gravity. Consequently, the wavelength of the instability, in units of $H$, increases. This is shown in Fig. 3 which plots the magnetic energies for $\Lambda_{0}=10$ and a range of $Q$ values. The number of vertical nodes decrease with $Q$, i.e. the disk accommodates fewer wavelengths because increasing vertical self-gravity makes it thinner.

We repeated the $\Lambda_{0}=10$ case with a stronger field $\beta=$ 25, shown in Fig. 2 as the dashed-triple dot line. Here, strong self-gravity is effective in reducing the growth rate, because decreasing $\beta$ enhances the dependence of $\lambda / H$ on $f(Q)$. For $Q=0.2$ and $\beta=25$ we find $\lambda_{\text {ideal }} / 2 H \sim 1$ at the midplane and the growth rate is reduced significantly.

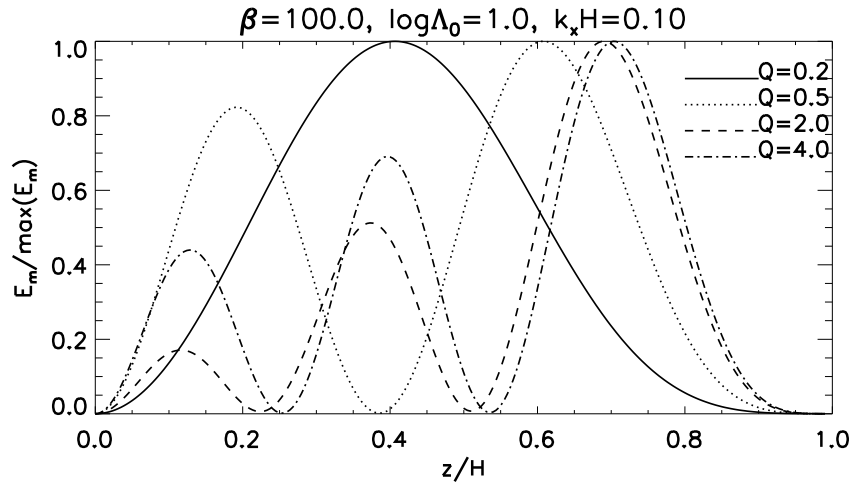

Figure 3. MRI magnetic energies in ideal polytropic disks for different strengths of self-gravity.

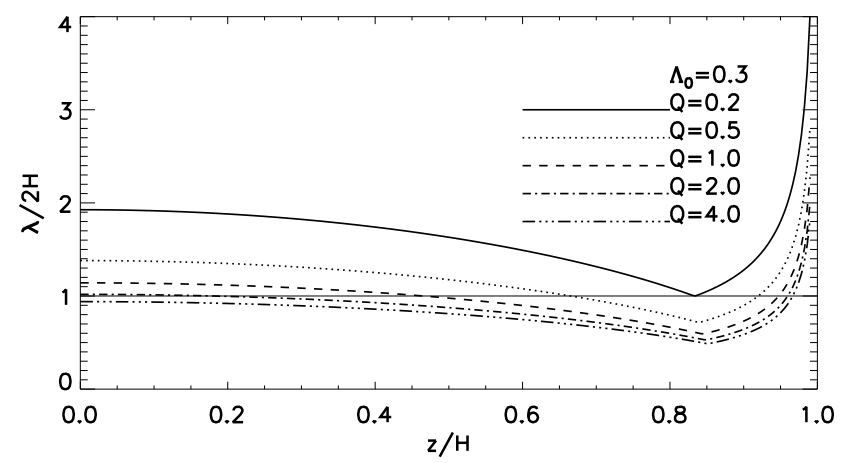

Figure 4. Approximate wavelengths of the most unstable MRI modes as given by Eq. 44 [46 normalized by the disk thickness, as a function of height. MRI is expected to operate if $\lambda / 2 H \lesssim 1$.

Self-gravity also appreciably decreases the MRI growth rates in the resistive limit. Fig. 4 plots Eq. 44 for $\Lambda_{0}=0.3$. In the non-self-gravitating disk $(Q=4)$ the instability criterion is marginally satisfied and the MRI operates. As $Q$ decreases, Eq. 44 is violated and the MRI growth rate is significantly reduced. This is seen for $Q=0.2$ where $\lambda \geq 2 H$ throughout the disk. (The instability is not suppressed since Eq. 45, 46 is only exact for unstratified disks.) Although the function $f(Q)$ does not change significantly for the range of $Q$ considered, the dependence of $\lambda / H$ on $f(Q)$ is amplified by the denominator $\Lambda_{0}<1$ in the resistive case. Modes in Fig. 4 have no nodes in the magnetic energy $E_{m}$ except at $z \simeq 0, H$, i.e. only the longest wavelength survives against large resistivity.

\subsubsection{Layered resistivity}

Here we consider disks with midplane Elsasser number $\Lambda_{0}=0.1$ and a variable resistivity profile with $A=10^{2}$. Fig. 5] compares the magnetic energies for $Q=0.2,1$ and 4 . They have similar growth rates, $\gamma / \Omega=0.53,0.64$ and 0.66 , respectively. In the non-self-gravitating limit $(Q=4)$, the MRI is effectively suppressed for $z \lesssim 0.5 H$. This is consistent with the picture of layered accretion proposed for non-self-gravitating disks Gammie 1996a; Fleming \& Stone 2003). However,in the massive disk $(Q=0.2)$ the mode occupies a wider vertical extent because its wavelength (in units of $H$ ) is larger. This suggests that in massive disks, the MRI is not well localized to a sub-layer within the height, even when the resistivity has a layered structure. 


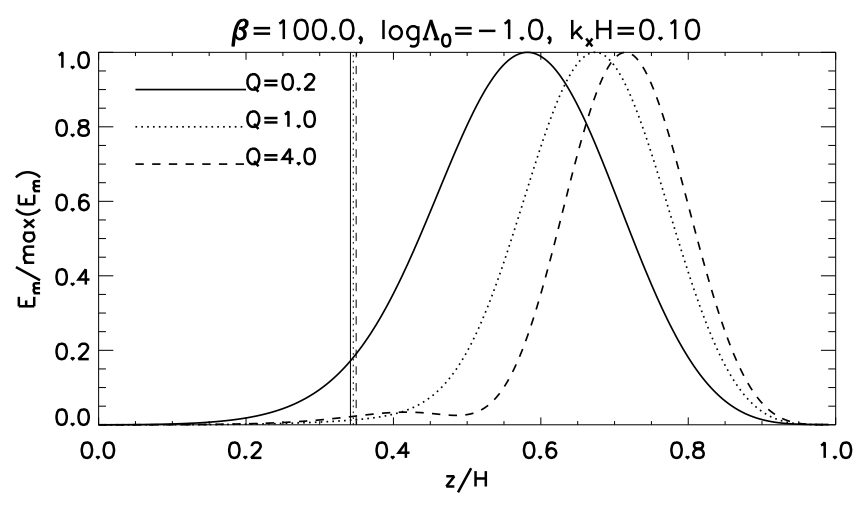

Figure 5. Magnetic energies as a function of height, for polytropic disks in which the conductivity increases by a factor $A=10^{2}$ in going from the midplane to the upper disk boundary. The vertical lines indicate $\Lambda=1$ for each value of $Q$.

We also performed additional calculations with $A=$ $10^{3}$ and $A=10^{4}$ (see Fig. 1 of Gressel et al. 2012). For $\Lambda_{0}=0.1$, we find no significant increase in the magnetic energy in the resistive zones. However, lowering $\Lambda_{0}$ gives similar results to Fig. [5] e.g. for $A=10^{3}$ and $\Lambda_{0}=10^{-2}$ or $A=10^{4}$ and $\Lambda_{0}=10^{-3}$ the magnetic energy penetrates into the resistive zone for strongly self-gravitating disks. In general, the magnetic energy density maximum moves toward the midplane with increasing self-gravity.

\subsubsection{Dependence on $k_{x}$}

The above experiments show that with increasing disk self-gravity, the MRI becomes more global in the vertical direction. We find a similar result in the horizontal direction. Fig. 6] show MRI growth rates as a function of $k_{x}$ for a range of $Q$ values. Increasing self-gravity decreases the cut-off radial wavenumber for the MRI. We checked that these modes have negligible density perturbations. Then we can understand this result by invoking the instability criteria for incompressible MRI in an unstratified Keplerian disk,

$$
v_{A}^{2}\left(k_{z}^{2}+k_{x}^{2}\right)<3 \Omega^{2}
$$

where $k_{z}$ is a vertical wavenumber (Kim \& Ostriker 2000). Setting $k_{z}^{2} \sim \Omega^{2} / v_{A}^{2}$ and non-dimensionalizing, we find

$$
k_{x} H \lesssim \frac{\sqrt{\beta}}{f},
$$

where order-unity factors have been dropped. Despite a simplistic approach, this demonstrates that with increasing self-gravity (increasing $f$ ), we expect MRI modes with small radial length scales to be suppressed.

\subsection{Influence of self-gravity on the MRI through the linear response}

Our goal here is to examine whether or not selfgravity can amplify the density perturbations associated with the MRI. We compute unstable modes in a massive isothermal disk with $Q=0.2$ (corresponding to $Q_{2 \mathrm{D}}=0.72$ ), which is still expected to be marginally stable to gravitational instability Mamatsashvili \& Rice 2010, who find a critical value of $Q \simeq 0.2$ ). The upper disk boundary is set to $Z_{s}=H$.

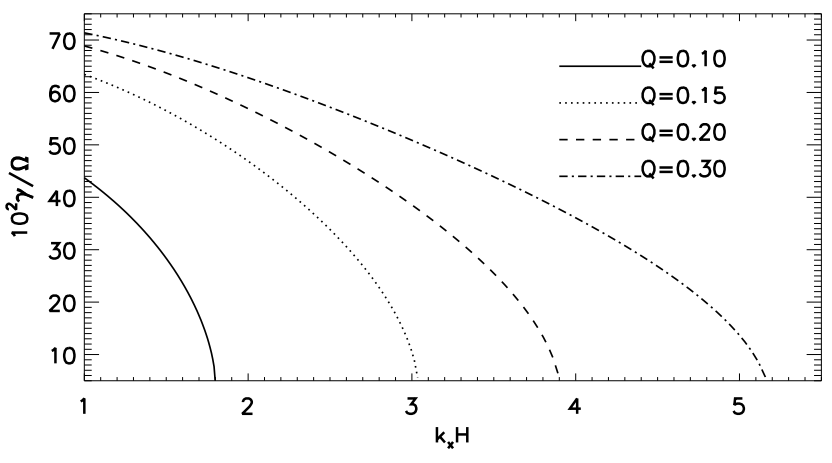

Figure 6. MRI growth rates in self-gravitating polytropic disks, as a function of the horizontal wavenumber $k_{x}$. The disk is ideal $\left(\Lambda_{0}=10^{2}, A=1\right)$ with $\beta=40$. These modes have negligible density/potential perturbations.

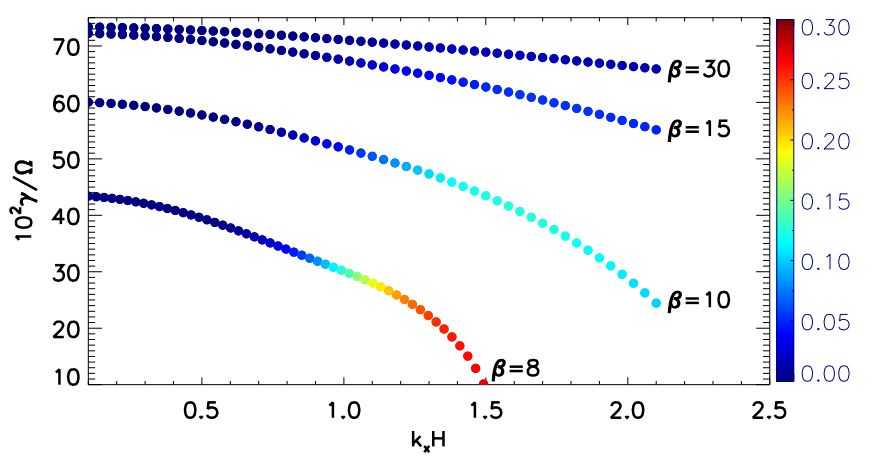

Figure 7. Growth rates of MRI modes in isothermal selfgravitating disks with $Q=0.2\left(Q_{2 \mathrm{D}}=0.72\right)$ in the limit of ideal $\operatorname{MHD}\left(\Lambda_{0}=10^{2}, A=1\right)$, for a range of field strengths $\beta$. The colorbar measures the importance of self-gravity by $\tau$.

\subsubsection{Ideal disks}

We first consider ideal MHD by adopting a uniform resistivity with $\Lambda_{0}=100$. Fig. 7 plots MRI growth rates as a function of $k_{x}$ for several values of $\beta$. The curves are color-coded according $\tau$. (Recall $\tau \rightarrow 1$ implies self-gravity dominates over magnetic perturbations, and $\tau \rightarrow 0$ is the opposite limit.) The potential perturbation is negligible for all cases when $k_{x} H \lesssim 0.5$, since the MRI becomes incompressible as $k_{x} \rightarrow 0$.

For $\beta \gg 1$, i.e. a weak field, density perturbations are negligible and the incompressible MRI operates. However, as $\beta$ is lowered and the MRI growth rate reduced, we find non-negligible potential perturbation for $k_{x} H=O(1)$. This suggests that in a strongly magnetized disk that still permits the MRI, the associated density perturbation can be important when the disk is self-gravitating.

\subsubsection{Resistive disks}

We repeat the above calculation for resistive disks, but fix $\beta=100$ and vary the midplane Elsasser number $\Lambda_{0}$. Growth rates are shown in Fig. 8. Interestingly, the highly resistive case $\Lambda_{0}=0.1$ has comparable magnetic and gravitational energies: at $k_{x} H \simeq 1.3$ we find $\tau \sim 0.3$, which corresponds to $\left\langle E_{g}\right\rangle \sim 0.5\left\langle E_{m}\right\rangle$. Fig. 9] compares the magnetic energy of this mode to that computed in the Cowling approximation, where the Poisson equation is ignored in the linearized equations and the potential 


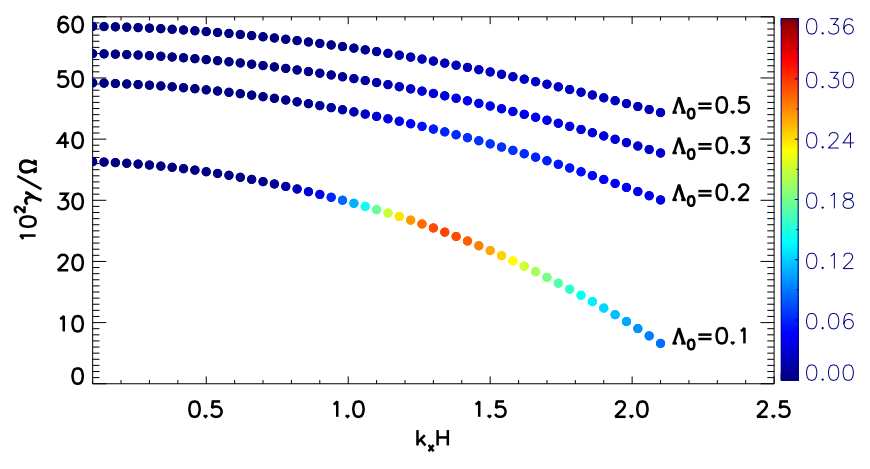

Figure 8. Growth rates of MRI modes in an isothermal selfgravitating disk with $Q=0.2\left(Q_{2 \mathrm{D}}=0.72\right)$ at fixed $\beta=100$, for a range of midplane Elsasser numbers. The resistivity is uniform. The colorbar measures the importance of self-gravity by $\tau$.

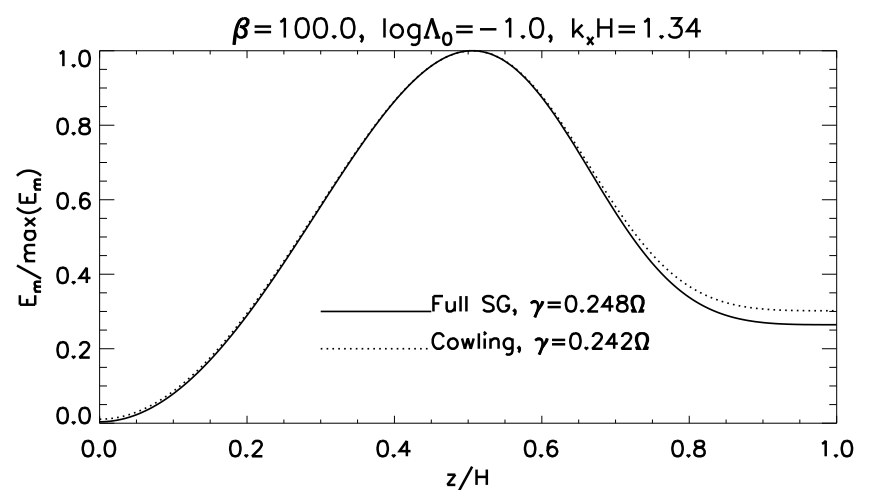

Figure 9. Magnetic energy associated with the linear mode with largest gravitational-to-magnetic energy ratio in Fig. 8 (solid) compared with that computed under the Cowling approximation (dotted).

perturbation set to zero (formally letting $Q \rightarrow \infty$ in Eq. 24). The growth rate increases when self-gravity is included in the linear response, since self-gravity is usually destabilizing. However, $\gamma$ and $E_{m}$ are very similar, indicating that the instability in the self-gravitating calculation is fundamentally still the MRI.

Fig. 10 plots the energies associated with the MRI mode discussed above. The gravitational energy exceeds the magnetic energy near the midplane $(z \lesssim 0.2 H)$. The growth rate $\gamma=0.25 \Omega$ is not much smaller than that of the most unstable mode $\left(\gamma=0.36 \Omega\right.$ for $\left.k_{x} H=0.1\right)$, so significant density perturbations will grow on dynamical timescales for this system, even though GI is not expected.

\subsubsection{Qualitative interpretation}

To make sense of the above results, we first return to ideal MHD and consider regions close to the disk midplane $(z \sim 0)$, where self-gravity is expected to be most important. For this discussion we will ignore stratification and set $d^{2} / d z^{2} \rightarrow-k_{z}^{2}$. The governing equations are then

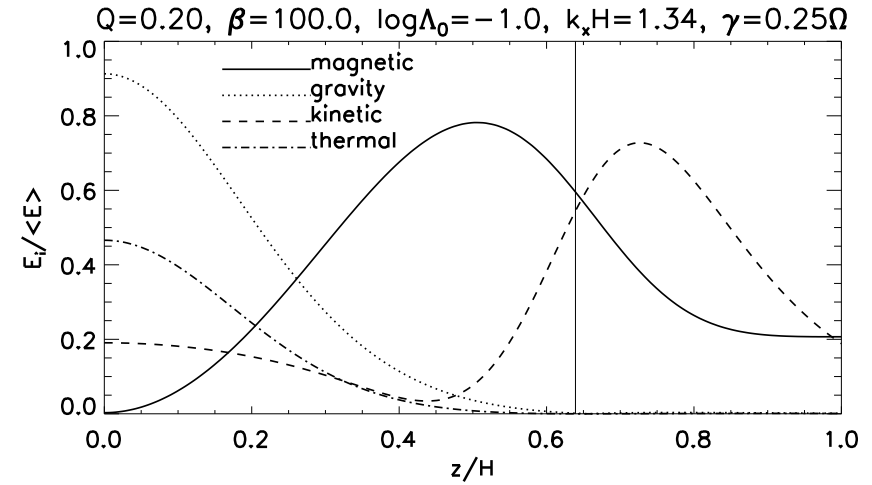

Figure 10. Example of a resistive MRI mode with significant gravitational potential perturbation. The disk is isothermal. The vertical line indicates $\Lambda=1$.

$$
\begin{aligned}
& 0=v_{A}^{2} k^{2} \delta v_{x}+\mathrm{i} \sigma\left(\mathrm{i} \sigma \delta v_{x}-2 \Omega \delta v_{y}+\mathrm{i} k_{x} \widetilde{W}\right) \\
& 0=v_{A}^{2} k_{z}^{2}\left(\delta v_{y}+\frac{\mathrm{i} S}{\sigma} \delta v_{x}\right)+\mathrm{i} \sigma\left(\mathrm{i} \sigma \delta v_{y}+\frac{\kappa^{2}}{2 \Omega} \delta v_{x}\right) \\
& 0=-k_{z}^{2} \widetilde{W}+\frac{\sigma^{2}}{c_{s}^{2}} W+\sigma k_{x} \delta v_{x} \\
& 0=k^{2} \delta \Phi+\frac{\Omega^{2}}{c_{s}^{2} Q} W
\end{aligned}
$$

where $k^{2}=k_{z}^{2}+k_{x}^{2}$. We imagine an iterative procedure to solve the above equations, starting from the Cowling approximation where $\delta \Phi \rightarrow 0$ and $Q \rightarrow \infty$. This is the standard MRI and we denote the solution as $\delta v_{x}^{(0)}, \delta v_{y}^{(0)}$ and $W^{(0)}$. Eq. 51 implies

$$
W^{(0)}=\frac{c_{s}^{2} \sigma k_{x} \delta v_{x}^{(0)}}{c_{s}^{2} k_{z}^{2}-\sigma^{2}} .
$$

We argue below that $c_{s}^{2} k_{z}^{2} \gg \sigma^{2}$ by taking $k_{z} \sim \Omega / v_{A}$. Then, recalling $W=c_{s}^{2} \delta \rho / \rho$, we can write

$$
\frac{\delta \rho^{(0)}}{\rho} \sim \frac{\sigma}{\Omega} \frac{1}{\beta}\left[\frac{k_{x} \delta v_{x}^{(0)}}{\Omega}\right] .
$$

The MRI has, in general, a non-zero density perturbation. However, it is negligible for $k_{x} \rightarrow 0$ and/or a weak field $(\beta \gg 1)$.

We now include self-gravity. The Poisson equation implies $W^{(0)}$ has an associated potential perturbation,

$$
\delta \Phi=-\frac{\Omega^{2}}{c_{s}^{2} Q k^{2}} W^{(0)} .
$$

Physically, we expect $k^{2} \geq 0$, so that a positive (negative) local density perturbation causes a negative (positive) local potential perturbation. We then insert $\delta \Phi$ back into the momentum and continuity equations, and ask how does this potential perturbation modify the Cowling solution? Writing $\delta v_{x}^{(0)} \rightarrow \delta v_{x}^{(0)}+\delta v_{x}^{(1)}$ and similarly for $\delta v_{y}$ and $W$, we find 


$$
\begin{aligned}
& k_{x} \sigma \delta \Phi=v_{A}^{2} k^{2} \delta v_{x}^{(1)}+\mathrm{i} \sigma\left[\mathrm{i} \sigma \delta v_{x}^{(1)}-2 \Omega \delta v_{y}^{(1)}+\mathrm{i} k_{x} W^{(1)}\right], \\
& 0=v_{A}^{2} k_{z}^{2}\left[\delta v_{y}^{(1)}+\frac{\mathrm{i} S}{\sigma} \delta v_{x}^{(1)}\right]+\mathrm{i} \sigma\left[\mathrm{i} \sigma \delta v_{y}^{(1)}+\frac{\kappa^{2}}{2 \Omega} \delta v_{x}^{(1)}\right], \\
& k_{z}^{2} \delta \Phi=\left(\frac{\sigma^{2}}{c_{s}^{2}}-k_{z}^{2}\right) W^{(1)}+\sigma k_{x} \delta v_{x}^{(1)} .
\end{aligned}
$$

Now, if the perturbations to the magnetic field remain unchanged, i.e. the mode remains close to the standard MRI as observed in Fig. 9, then $\delta v_{x}^{(1)} \sim 0$ and $\delta v_{y}^{(1)} \sim 0$, so Eq. 57 is satisfied. Eq. 56 then require $\delta \Phi+W^{(1)} \sim 0$. This is compatible with Eq. 58 if

$$
\left|k_{z}^{2}\right| \gg\left|\frac{\sigma^{2}}{c_{s}^{2}}\right| \text {. }
$$

For the ideal MRI, we take $k_{z} \sim \Omega / v_{A}$. Then $\left|\sigma^{2} / c_{s}^{2} k_{z}^{2}\right| \sim$ $\left|\sigma^{2} / \Omega^{2} \beta\right| \ll 1$ because $|\sigma| \lesssim \Omega$ and we are considering $\beta \gtrsim 10$. Thus Eq. 59 is generally satisfied.

The above assumptions imply

$$
W^{(1)} \sim \frac{\Omega^{2}}{c_{s}^{2} Q k^{2}} W^{(0)},
$$

which indicates a non-zero density perturbation due to the MRI can be amplified by self-gravity. Now, for $k_{x} H \sim 1$ we have $\left|k_{z}^{2} / k_{x}^{2}\right| \sim \beta / f^{2} \gg 1$ because $f=O(1)$ and $\beta \gtrsim 10$ for the cases considered above. Then

$$
\left|\frac{W^{(1)}}{W^{(0)}}\right| \sim \frac{1}{Q \beta},
$$

suggesting stronger amplification of the density field by self-gravity with increasing field strength (decreasing $\beta$ ).

The above arguments can be adapted to the resistive disk. Eq. [51 52 are unchanged, while resistive terms appearing in Eq. 49 50 only involve the potential perturbation through $\widetilde{W}$. For the resistive MRI we take $k_{z} \sim v_{A} / \eta$ and $|\sigma| \sim v_{A}^{2} / \eta=\Lambda \Omega$ (Sano \& Miyama 1999). Then $\left|\sigma^{2} / c_{s}^{2} k_{z}^{2}\right| \sim 1 / \beta \ll 1$ so Eq. 59 is satisfied. Noting that $k_{z}^{2} \sim \Lambda^{2} \Omega^{2} \beta / c_{s}^{2}$, the feedback equation becomes

$$
\left|\frac{W^{(1)}}{W^{(0)}}\right| \sim \frac{1}{Q\left(f^{2} \hat{k}_{x}^{2}+\beta \Lambda^{2}\right)},
$$

so increasing the resistivity (decreasing $\Lambda$ ) should enhance density perturbations.

For weak fields in an ideal disk, the MRI has a vertical wavelength $\lambda \ll H$. It will be almost incompressible so the 'seed' density perturbation $W^{(0)}$ is small. The perturbed mass contained within $\sim \lambda$ is small and its potential is unimportant. Furthermore, considering the stratified disk, $\lambda \ll H$ imply rapid variations in the density perturbation across the disk height, averaging to zero, so the magnitude of the associated potential perturbation is small. Self-gravity does not affect the MRI in this regime.
However, a strong field and/or large resistivity increases the MRI vertical wavelength. When the vertical scale of the MRI becomes comparable to the disk thickness, i.e. $\lambda \sim H$, the perturbed mass across the disk height can contribute to a net potential perturbation. We therefore expect a necessary condition for self-gravity to affect the MRI is for the latter to be weak.

\section{GRAVITATIONALLY UNSTABLE DISKS}

Gravitational instability becomes possible in a sufficiently massive and/or cold disk. Here, we explore whether or not GI and MRI can interact by computing unstable modes for isothermal disks with $Q<0.2$ $\left(Q_{2 \mathrm{D}} \lesssim 0.67\right)$ which permits GI, as shown below. We consider ideal disks with $\Lambda_{0}=100$ and $A=1$, unless otherwise stated.

\subsection{Co-existence of MRI and GI}

Fig. 11 show growth rates for modes with $k_{x} H=1$ as a function of $\beta$ in disks with $Q=0.18, Q=0.14$ and $Q=0.12$. All three cases display distinct GI modes (red/brown branch). The GI growth rates are $\gamma \simeq 0.25 \Omega, 0.6 \Omega, 0.8 \Omega$ for $Q=0.18,0.14,0.12$, respectively. GI is stabilized by magnetic pressure for sufficiently small $\beta$. The critical field strength for stabilizing GI increases with increasing self-gravity, consistent with Nakamura (1983). For $Q=0.18$, GI is stabilized for $\beta \lesssim 15$. Nevertheless, the MRI branch for $\beta<15$ becomes self-gravitating, so that density perturbations still grow, even though GI does not formally operate.

The GI and MRI branches only interact when their growth rates are similar. This is seen in Fig. 11 for $Q=0.18$ where the GI branch approaches a MRI branch at $\beta \simeq 25, \gamma \simeq 0.2 \Omega$. In fact, following the red curve to smaller $\beta$ indicates GI transitions to MRI. The 'gaps' in the GI and MRI branches for $Q=0.18$ and $Q=0.12$ may be due to the phenomenon of avoided crossing, as seen in stars (e.g. Aizenman et al. 1977) and accretion tori/disks (e.g. Christodoulou 1993; Ogilvie 1998), where physically distinct modes approach one another in frequency and exchange character. However, we cannot exclude the possibility that some modes may have been missed in a numerical search of eigenfrequencies.

Thus, our results do not rigorously prove that the GI and MRI branches do not intersect. Nevertheless, the continuous variation of $\tau$ strongly suggest that unstable modes can transition smoothly from MRI to GI and vice versa, especially at low $\beta$.

\subsubsection{Case study}

In reality, perturbations with a range of $k_{x}$ will be present for a given set of disk parameters. Fig. 12 show growth rates as a function of $k_{x}$ in a disk with $Q=0.12, \beta=20$, where MRI and GI have comparable growth rates. All perturbations with $k_{x} H \lesssim 3.5$ grow dynamically $(\gamma \gtrsim 0.1 \Omega$, or $\lesssim 1.6$ orbits $)$.

We also plot in Fig. 12 growth rates obtained from the Cowling approximation, which isolates MRI; and that from a high-resistivity run, which isolates GI by allowing the field lines to slip through the fluid. We refer to these as pure MRI and pure GI, respectively. For $k_{x} H \lesssim 0.7$, growth rates are equal to those on the pure MRI and pure GI branches. That is, MRI and GI operate independently until their growth rates become equal as a function of $k_{x}$. 


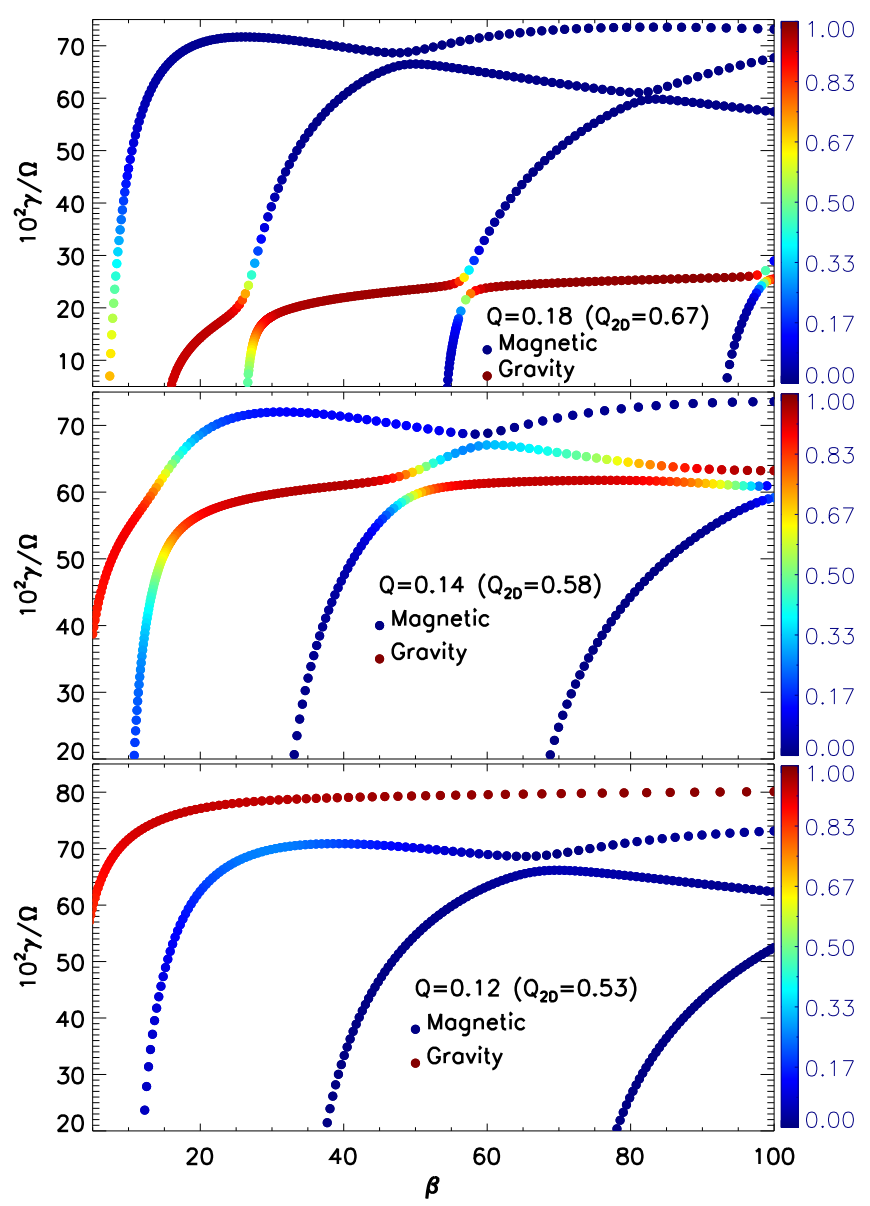

Figure 11. Growth rates for modes with $k_{x} H=1$ in isothermal ideal disks with $Q=0.18$ (top), $Q=0.14$ (middle) and $Q=0.12$ (bottom). The colorbar measures the importance of self-gravity by $\tau$

The dispersion relation $\gamma\left(k_{x}\right)$ deviates from the pure GI/MRI curves with increasing $k_{x}$, implying stronger interaction between magnetic and density perturbations. Comparing pure GI (dashed line) and the gravitationallydominated portions of $\gamma\left(k_{x}\right)$ shows that inclusion of magnetic field stabilizes high- $k_{x}$ pure GI. (Note also the slight decrease in the most unstable $k_{x}$.) This stabilization is due to magnetic pressure (Lizano et al. 2010), consistent with pressure stabilizing small-wavelength GI only.

Comparing pure MRI (solid line) and the magneticallydominated portions of $\gamma\left(k_{x}\right)$ show that self-gravity increases MRI growth rates at large $k_{x}$. This effect is small but noticeable, which can be used as a code test for non-linear simulations. Note that this destabilization by self-gravity is through the linear response, rather than through the background stratification (which is stabilizing).

\section{EFFECT OF AN AZIMUTHAL FIELD}

In this section we use the setup of case 2 described in 93. and examine the effect of an azimuthal field so that $B_{y} \neq 0$, parametrized by $\epsilon \equiv B_{y} / B_{z}$. However, we continue to use $B_{z}$ for normalizations and $\beta$ is associated with the vertical Alfven speed. We also extend the previous calculations to the full disk $z \in\left[-Z_{s}, Z_{s}\right]$, which allows us to compare the effect of self-gravity on MRI modes with different symmetries across the midplane.

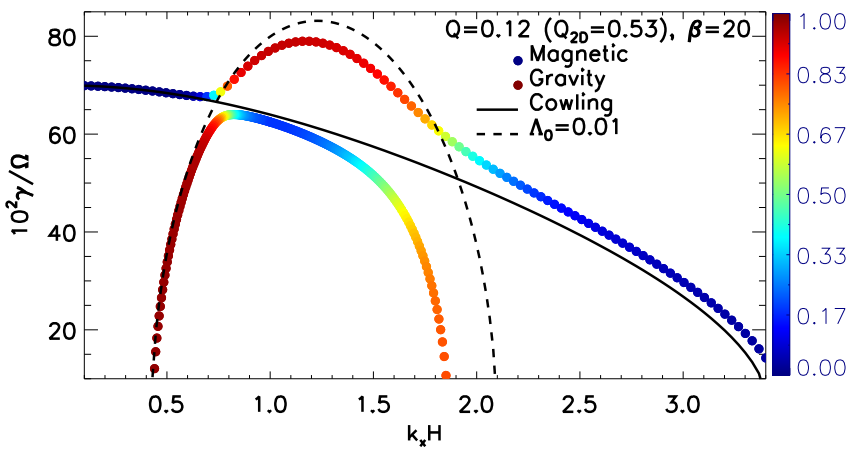

Figure 12. Growth rates of unstable modes in the massive isothermal disk with $Q=0.12$ and $\beta=20$, as a function of the horizontal wavenumber $k_{x}$. The colorbar measures the importance of self-gravity by $\tau$. The solid line corresponds to MRI modes in the Cowling approximation. The dashed line corresponds to pure GI modes, obtained by including a high resistivity in the full problem.

We use an isothermal disk throughout.

\subsection{Ideal disks with MRI}

We consider disks with $Q=0.2\left(Q_{2 \mathrm{D}}=0.72\right)$ and $\beta=$ 10 in the limit of ideal MHD $\left(\Lambda_{0}=100\right)$. Gravitational instability is not expected because Fig. 11] shows that even for $Q=0.18$, GI is suppressed for $\beta \lesssim 15$.

Fig. 13 show MRI growth rates for $B_{y} / B_{z}=0,1,2$ and 3 . We divide the modes into two categories depending on the extremum of magnetic energy at the midplane. The top panel are modes where $E_{m}$ has a local minimum at $z=0$ and the bottom panel are modes where $E_{m}$ has a local maximum at $z=0$. The latter set of modes were excluded in the previous sections by midplane boundary conditions. We also plot growth rates computed in the Cowling approximation. As expected, $\left\langle E_{g}\right\rangle<\left\langle E_{m}\right\rangle$, so none of the modes are energetically dominated by selfgravity.

Consider first modes in the top panel of Fig. 13. As with previous results, self-gravity destabilizes modes with $k_{x} H \gtrsim O(1)$. Consequently, the cut-off wavenumber is larger when SG is included. Destabilization is most effective for purely vertical fields: with $\epsilon=0, k_{x} H \simeq 1.4$, $\mathrm{SG}$ increases the growth rate by $\sim 30 \%$. For $B_{y}=0$ we find the density perturbation $W(z)$ is an even function. Although these modes are fundamentally magnetic, this is consistent with Goldreich \& Lynden-Bell (1965a), who showed that SG can only destabilize symmetric density perturbations. With increasing $B_{y}$, we find $W$ deviates from an even function. Together with the increased total magnetic pressure with $B_{y}$ (since $B_{z}$ is fixed), destabilization by SG weakens. Thus, the Cowling approximation becomes increasingly good with stronger $B_{y}$ for these modes.

The modes in the bottom panel of Fig. 13 display opposite behavior. For $B_{y}=0$ we find $W(z)$ is odd, and self-gravity has no effect. When $B_{y}>0, W$ deviates from an odd function and the midplane density perturbation $|W(0)|$ increases. SG is stabilizing for these modes at all wavelengths, and is most effective at $k_{x} H=O(1)$. Fig. 14 show eigenfunctions for $\epsilon=3$ and $k_{x} H=1.1$ with and without the Cowling approximation. SG significantly enhances the midplane density perturbation, making the gravitational potential energy comparable to 


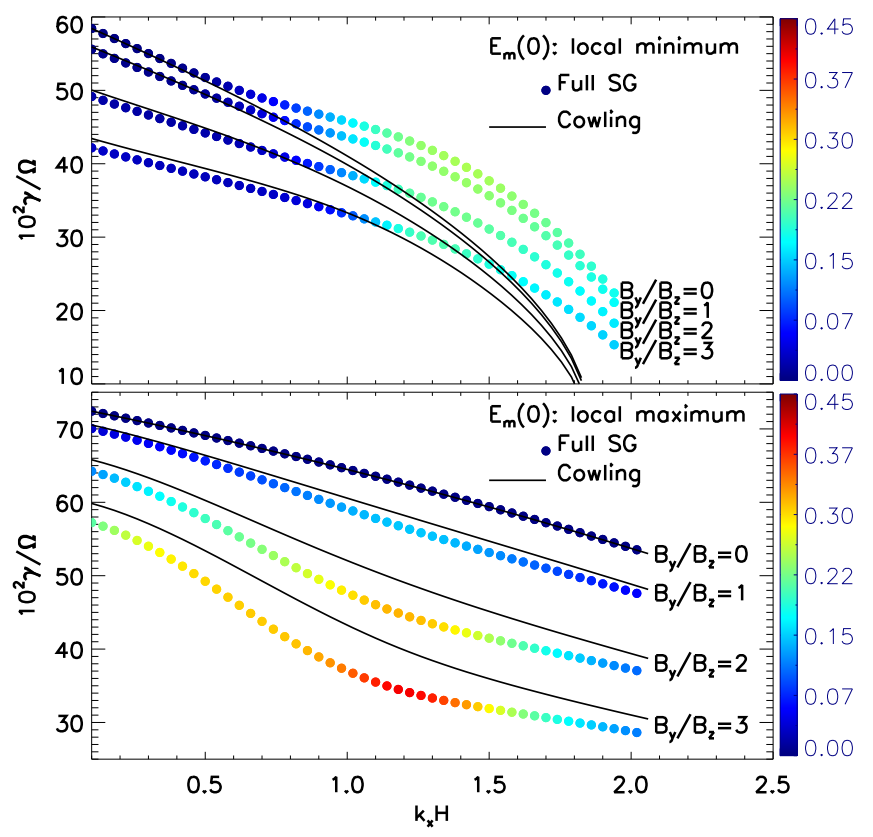

Figure 13. MRI growth rates in isothermal disks with $Q=0.2$ $\left(Q_{2 \mathrm{D}}=0.72\right)$ and $\beta=10$ for a range of azimuthal field strengths $B_{y} / B_{z}$. The dots are solutions computed from the full problem, with the colorbar measuring the gravitational potential perturbation via $\tau$, while the solid curves are computed from the Cowling approximation. For $B_{y}=0$, modes in top and bottom panels have $W^{\prime}(0)=0$ and $W(0)=0$, respectively.
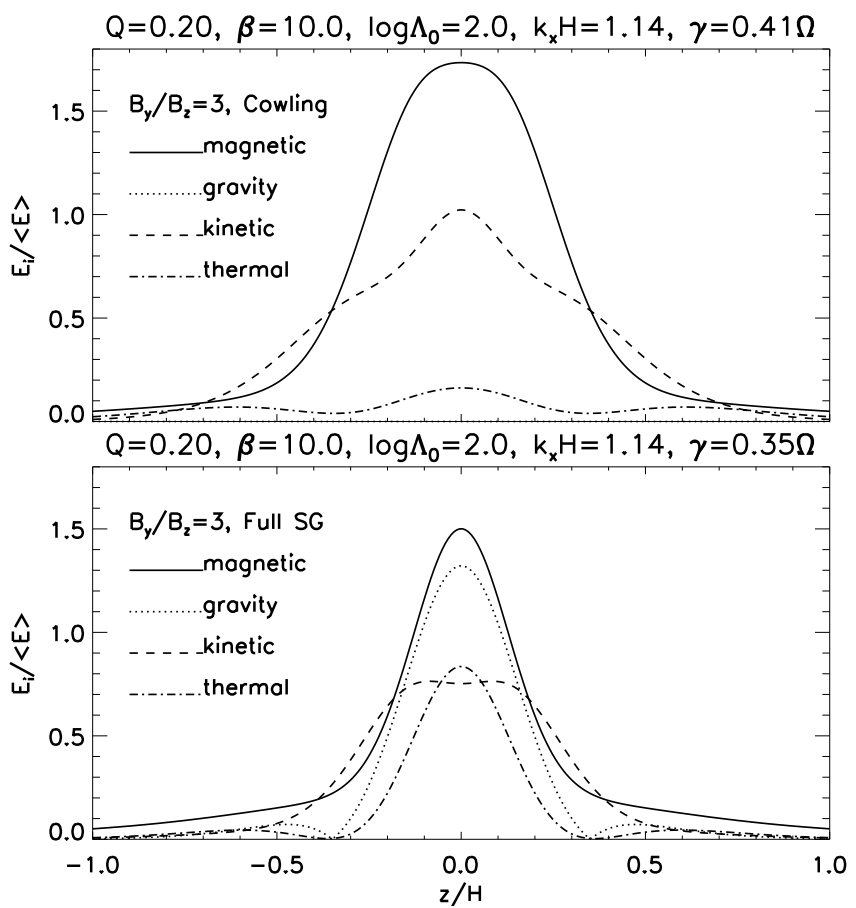

Figure 14. Energy densities for a MRI mode in an isothermal ideal disk with an azimuthal field $B_{y}=3 B_{z}$, computed in the Cowling approximation (top) and with full self-gravity (bottom). These modes correspond to those in the bottom panel of Fig. 13

the magnetic energy, which becomes more confined near the midplane.

To interpret the above result for modes with magnetic energy concentrated at the midplane, we note that compressibility affects the MRI in the presence of an az- imuthal field even in a non-self-gravitating disk. If the perturbed disk remains in vertical hydrostatic equilibrium, then

$$
|W| \sim \frac{B_{y}}{\mu_{0} \rho}\left|\delta B_{y}\right|,
$$

to order of magnitude in a non-SG disk. Thus a strong azimuthal field can cause a large density perturbation (Pessah \& Psaltis 2005). We checked that for the modes in Fig. 14, vertical velocities are small, $\left|\delta v_{z}\right| /\left(\left|\delta v_{x}\right|^{2}+\left|\delta v_{y}\right|^{2}\right)^{1 / 2} \lesssim 0.2$.

Compressibility is enhanced by an azimuthal field, which is stabilizing for the MRI (Kim \& Ostriker 2000). This effect is significant for $\epsilon=3$ because the azimuthal Alfven speed is sonic. Fig. 14 indicates that self-gravity further enhances compressibility, and therefore stabilization. We suspect this is overwhelmed by the destabilization effect of $\mathrm{SG}$, because the density perturbation has an anti-symmetric component.

\subsection{Resistive disks with GI}

Here we examine a resistive disk which permits MRI and GI by setting $Q=0.18, \Lambda_{0}=0.1$ and $\beta=100$. Fig. 15 show growth rates for $\epsilon=0,1$ and 2. For $B_{y}=0$, MRI and GI are decoupled except for a narrow range of $k_{x}$ in which the lower MRI modes transitions to GI. Notice that the upper MRI modes intersect the GI branch. There is no interaction because the upper MRI modes have anti-symmetric $W(z)$ whereas the GI modes have symmetric $W(z)$.

Introducing $B_{y}=B_{z}$ leads to an exchange in the mode characters. For $k_{x} H \lesssim 0.9$ the modes on the two MRI branches are similar to the vertical field case. However, for $k_{x} H \gtrsim 0.9$ the upper MRI mode transitions to GI, for which $E_{m}(0)$ is a minimum; and the lower MRI mode has $E_{m}(0)$ being a maximum. We find all perturbations with $k_{x} H \gtrsim 0.9$ have symmetric $W(z)$.

Increasing the azimuthal field further to $B_{y}=2 B_{z}$ we find overstable MRI modes with non-negligible real frequencies (Gammie 1996a). An example is shown in Fig. 16. Notice the density/potential perturbation is off-set from the midplane. This is not possible for pure GI (Goldreich \& Lynden-Bell 1965a). Thus, these overstable MRI modes indeed become self-gravitating, before being stabilized.

Notice also in Fig. 15 the disappearance of magnetic modes between $0.8 \lesssim k_{x} H \lesssim 1.5$ as $B_{y}$ is increased. For $B_{y}=2 B_{z}$, MRI and GI are again independent because they operate at distinct radial scales. This implies that perturbations unstable to GI cannot develop MRI.

\section{SUMMARY AND DISCUSSION}

In this paper, we have performed axisymmetric linear stability calculations of magnetized, self-gravitating, vertically stratified disks in the local approximation. Our models include resistivity and azimuthal fields. We have identified regimes under which the magneto-rotational instability (MRI) is affected by disk self-gravity (SG).

For a vertical field, the requirement for the MRI to operate is that its vertical wavelength $\lambda \lesssim 2 H$. The disk thickness $H=H(Q)$ decreases with increasing SG. This reduces MRI growth rates when $\beta$, and hence $\lambda$, is fixed. Thus, a sufficiently massive disk can potentially 


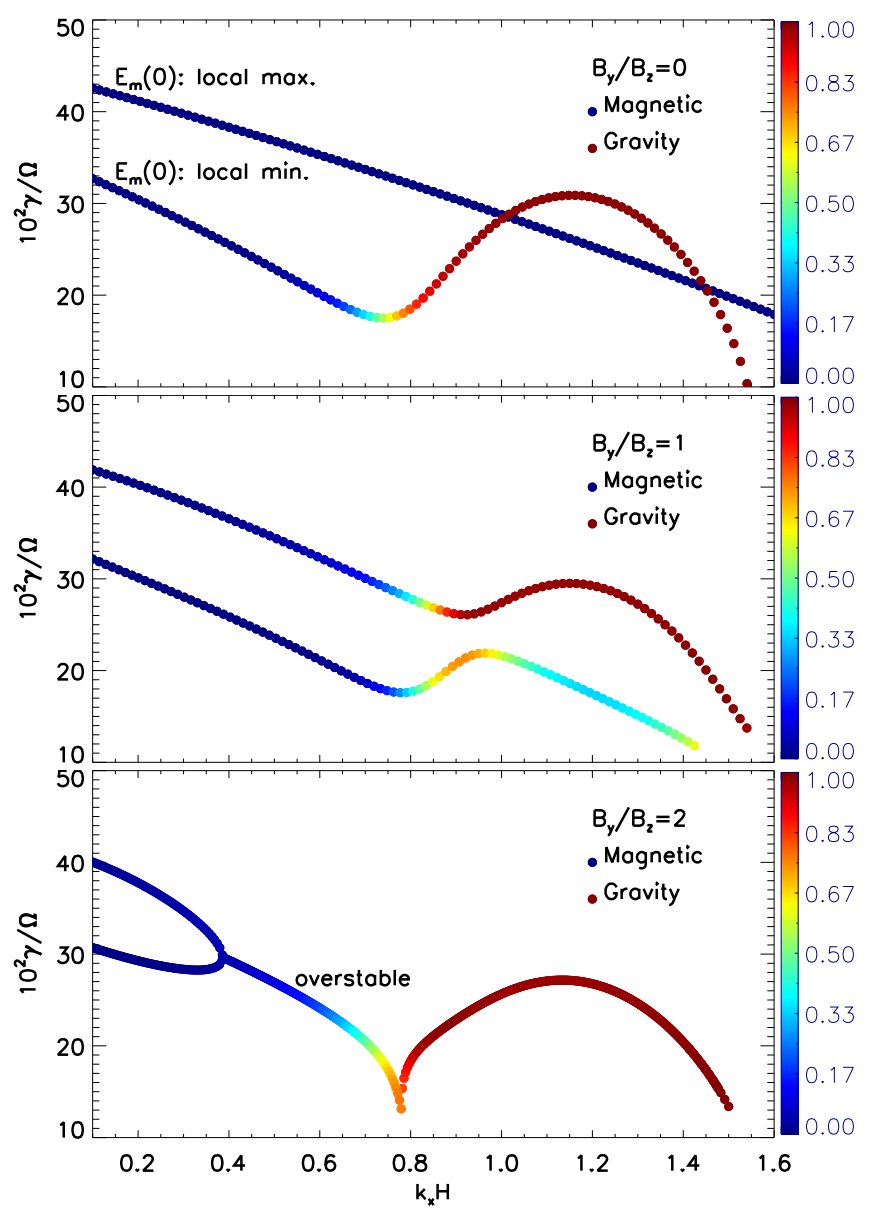

Figure 15. Growth rates in isothermal resistive disks with $Q=$ $0.18\left(Q_{2 \mathrm{D}}=0.67\right), \beta=100$ and $\Lambda_{0}=0.1$. For $B_{y}=0$, the upper and lower MRI modes have anti-symmetric and symmetric density perturbations, corresponding to $W(0)=0$ and $W^{\prime}(0)=0$, respectively. For $B_{y} / B_{z}=2$ the overstable modes have non-zero real frequencies.

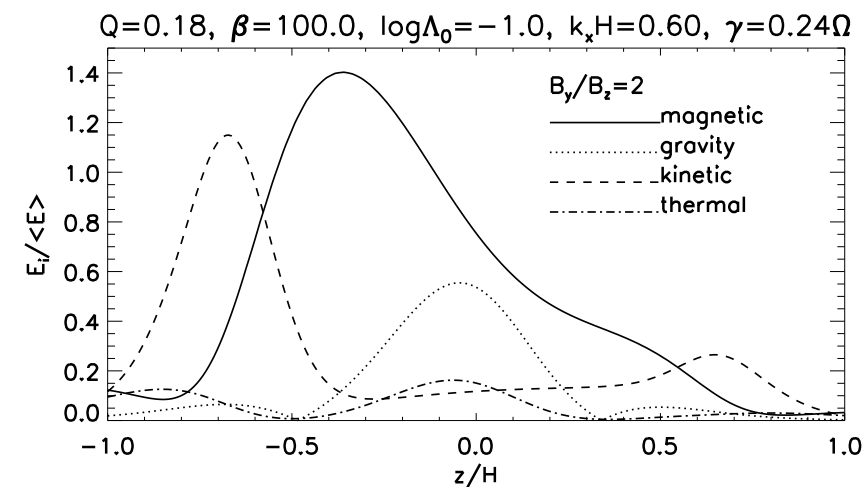

Figure 16. Overstable MRI mode in an isothermal resistive disk with $Q=0.18\left(Q_{2 \mathrm{D}}=0.67\right), \Lambda_{0}=0.1$ and $\beta=100$. The mode has a real frequency $\omega=0.059 \Omega$, or $\omega / \gamma \simeq 0.2$.

suppress the MRI. The MRI is also restricted to larger radial scales as $Q$ is lowered. This means that the MRI becomes more global in self-gravitating disks.

The condition $\lambda<2 H$ may be written more precisely as

$$
\frac{n}{\min \left(\Lambda_{0}, 1\right)} \frac{f(Q)}{\sqrt{\beta}} \lesssim 1
$$

where $n \sim 3$ is a numerical factor and $\min \left(\Lambda_{0}, 1\right)$ accounts for the ideal and resistive limits (see Eq. 4546). Since $f$ increases with decreasing $Q$, Eq. 64 implies the MRI requires larger values of $\beta$ with increasing selfgravity. For definiteness, consider the ideal polytropic disk. Then Eq. 64 is

$$
\beta^{-1 / 2} \lesssim \frac{\sqrt{15}}{4 \pi} \sqrt{Q} \arccos \left(\frac{Q}{1+Q}\right)
$$

For a non-self-gravitating disk, $Q \rightarrow \infty$ and Eq. 65] is $\beta \gtrsim 16 \pi^{2} / 30 \simeq 5$. For $Q \ll 1$, the condition is $\beta \gtrsim$ $64 / 15 Q$, giving $\beta \gtrsim 20$ for $Q=0.2$. We confirm this numerically, finding the MRI growth rate $\gamma \lesssim 0.1$ when $\beta \lesssim 3.3$ for $Q=20$ and $\beta \lesssim 17$ for $Q=0.2$.

We can also place an upper bound on the absolute field strength $B_{z}$. Writing $v_{A 0}=B_{z} \sqrt{4 \pi G Q / \mu_{0} \Omega^{2}}$, we find Eq. 65) is independent of $Q$ for $Q \ll 1$, and

$$
\frac{B_{z}}{c_{s 0} \Omega} \sqrt{\frac{\pi G}{\mu_{0}}} \lesssim \frac{\sqrt{15}}{16}
$$

is needed for the MRI to operate in the ideal polytropic disk with strong self-gravity. Although both the MRI wavelength and disk thickness vanish as $Q \rightarrow 0$, the MRI can still operate provided the field is sufficiently weak according to Eq. 66 .

Interestingly, for layered resistivity we do not find layered magnetic perturbations when the disk is massive. This is consistent with the MRI becoming vertically global with increasing self-gravity. For non-selfgravitating disks $\lambda \ll H$, so the MRI can be restricted to regions of size $L<H$, i.e. an active layer. This not compatible with $\lambda \sim H$, as found for massive disks. Hence we find magnetic perturbations penetrate into the high-resistivity dead zone (e.g. $Q=0.2$ in Fig. 5), and there is no distinct boundary between active and dead layers. This suggests that the picture of layered accretion (e.g. Fleming \& Stone 2003) may not be applicable to self-gravitating disks.

We find MRI modes with radial scales of $\sim H$ can acquire density perturbations in massive but Toomrestable disks. This occurs when the MRI is weak, for example with a strong field or high resistivity. We argue in that case $\lambda \sim H$, so the MRI is compressible and the associated density perturbation can be enhanced by self-gravity.

At this point it is worth mentioning previous nonlinear simulations of magnetized self-gravitating galactic and circumstellar disks (Kim et al. 2003; Fromang et al. 2004d, c). These authors find self-gravity did not enhance MRI density fluctuations significantly. However, they employed ideal MHD simulations with gas-to-magnetic pressure ratios of order $10^{2}$ to $10^{3}$. This is qualitatively consistent with our results, as self-gravity is not expected to influence the MRI in this regime of $\beta$, except through the background state. For example, Fromang et al. (2004c) found MRI turbulence is more coherent in selfgravitating disks. This may be related to our finding that 
small radial scale MRI is suppressed when self-gravity is included in the background equilibria.

Physically, we expect MRI to interact with self-gravity when their spatial scales are similar. Because self-gravity acts globally in the vertical direction, for it to affect the MRI, future non-linear simulations should consider parameter regimes in which the MRI is vertically global. Indeed, in the setup of Kim et al. (2003), the disk scale height exceeds the MRI vertical wavelength and selfgravity has little impact.

Curiously, when GI and MRI are simultaneously supported, we find unstable modes transition between MRI and GI. There exists modes with comparable potential and magnetic energy perturbations, which demonstrates MRI and GI can interact. These transitions occur smoother with decreasing $\beta$ (Fig. 11) or increasing $k_{x}$ (Fig. 12). The latter implies that, in order to capture the magneto-gravitational interactions represented by these intermediate modes, non-linear simulations must resolve radial scales smaller than the most unstable GI mode. For example, Fig. 12 suggest radial scales down to $\sim H / 2$ should be well-resolved.

We examined the effect of an additional azimuthal field, while keeping the vertical field at fixed strength. In this case, we also relaxed the equatorial symmetry condition applied previously and considered the full disk column. Self-gravity affects the MRI differently depending on its character. Self-gravity destabilizes MRI modes where the magnetic energy has a minimum at $z=0$, these modes have a symmetric density perturbation in the limit $B_{y} \rightarrow 0$. However, self-gravity stabilizes MRI modes where the magnetic energy has a maximum at $z=0$, these modes have an anti-symmetric density perturbation in the limit $B_{y} \rightarrow 0$. This stabilization effect is stronger for increasing $B_{y}$. Previous linear calculations show that increased compressibility associated with a toroidal field stabilizes the MRI (Kim et al. 2003). We conjecture that self-gravity further enhances this effect. Non-linear MRI simulations with strong toroidal fields that neglect self-gravity may over-estimate the strength of MRI turbulence.

\subsection{Caveats and outlooks}

\section{APPENDIX}

\section{ANALYTIC EQUILIBRIUM FOR THE POLYTROPIC DISK}

For a polytropic disk with $P=K \rho^{2}$ the dimensional equilibrium equation to be solved is

$$
0=c_{s 0}^{2} \frac{d^{2}}{d z^{2}}\left(\frac{\rho}{\rho_{0}}\right)+\Omega_{z}^{2}+\frac{\Omega^{2}}{Q}\left(\frac{\rho}{\rho_{0}}\right),
$$

which is obtained by combining Eq. [6] and 7 with the above equation of state. The solution is

$$
\frac{\rho}{\rho_{0}}=\left(1+\frac{\Omega_{z}^{2}}{\Omega^{2}} Q\right) \cos (a z)-\frac{\Omega_{z}^{2}}{\Omega^{2}} Q,
$$

where

$$
a^{2} \equiv \frac{\Omega^{2}}{Q c_{s 0}^{2}} \text {. }
$$

The polytropic disk thickness is

$$
H=\frac{c_{s 0}}{\Omega} \sqrt{Q} \arccos \left(\frac{\Omega_{z}^{2} Q}{\Omega^{2}+\Omega_{z}^{2} Q}\right) .
$$




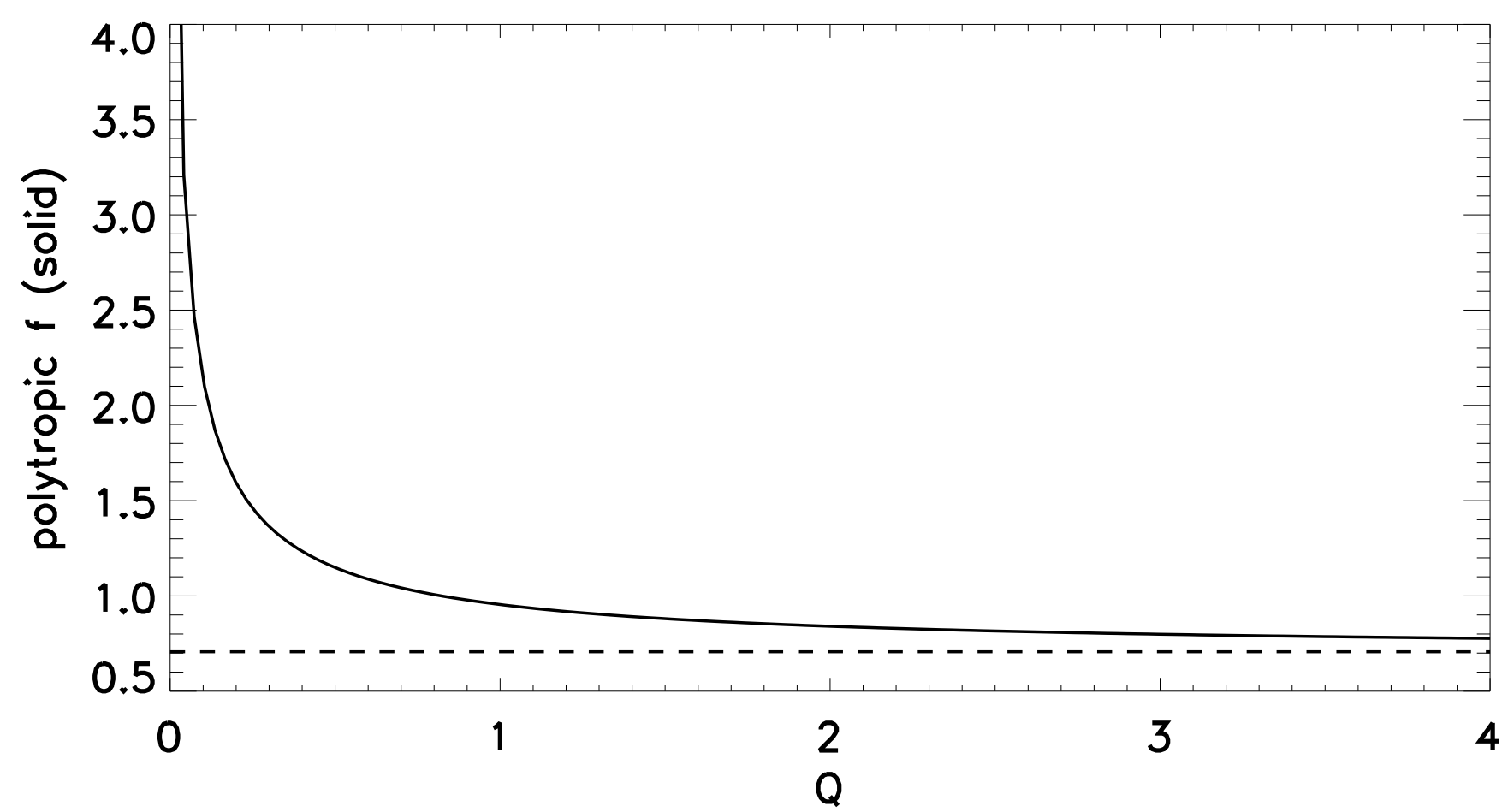

Figure 17. The function $f(Q)$ describing vertical hydrostatic equilibrium in self-gravitating polytropic disks (solid line). The horizontal dashed line is the asymptotic value of $1 / \sqrt{2}$ for large $Q$.

Given a fixed mid-plane temperature, the function $f(Q) \equiv c_{s 0} / \Omega H$ is an inverse measure of the disk thickness, and $f$ increases with decreasing $Q$, as shown in Fig. 17. This corresponds to a thinner disk with increasing strength of vertical self-gravity.

\section{RELATION BETWEEN $Q$ AND THE TOOMRE PARAMETER}

The Toomre parameter defined for razor-thin disks is

$$
Q_{2 \mathrm{D}} \equiv \frac{\kappa c_{s}}{\pi G \Sigma}
$$

where $\Sigma$ is the total column density. To relate our self-gravity parameter $Q$ and $Q_{2 \mathrm{D}}$, we replace $c_{s}$ by $\overline{c_{s}} \equiv$ $\int \rho c_{s} d z / \int \rho d z$, and $\kappa$ by $\Omega$, giving

$$
Q_{2 \mathrm{D}}=2 Q f \frac{\int_{0}^{1} \hat{\rho} \hat{c}_{s} d \hat{z}}{\left(\int_{0}^{1} \hat{\rho} d \hat{z}\right)^{2}},
$$

where each term on the right-hand-side is non-dimensionalized (see $\$ 3.2 .1$ ). Fig. 18 plots this relation for isothermal and polytropic disks.

\section{REDUCTION TO LINEAR HYDRODYNAMICS}

Our task here is to remove the magnetic field and vertical velocity perturbations from the linearized equations. Let us first define operators

$$
D_{0}=1, \quad D_{1}=\frac{\rho^{\prime}}{\rho}+\frac{d}{d z}, \quad D_{2}=\frac{\rho^{\prime \prime}}{\rho}+\frac{2 \rho^{\prime}}{\rho} \frac{d}{d z}+\frac{d^{2}}{d z^{2}},
$$

and

$$
\bar{D}_{0}=\eta D_{0}, \quad \bar{D}_{1}=\eta^{\prime} D_{0}+\eta D_{1}, \quad \bar{D}_{2}=\eta^{\prime \prime} D_{0}+2 \eta^{\prime} D_{1}+\eta D_{2} .
$$

And we define the variables

$$
\begin{aligned}
U & \equiv \mathrm{i} \sigma \delta v_{x}-2 \Omega \delta v_{y}+\mathrm{i} k_{x} \widetilde{W}, \\
V & \equiv \mathrm{i} \sigma \delta v_{y}+\frac{\kappa^{2}}{2 \Omega} \delta v_{x} .
\end{aligned}
$$




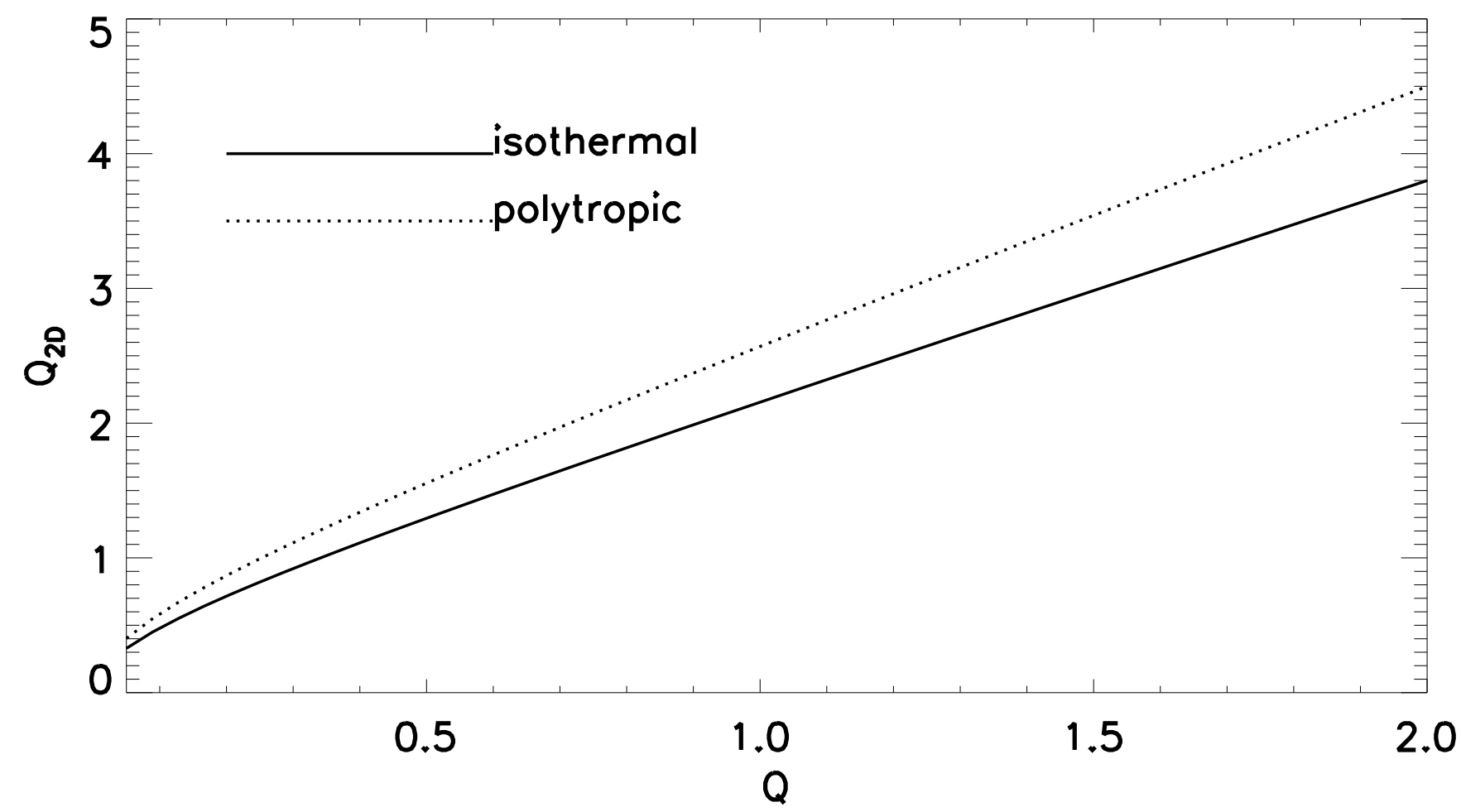

Figure 18. Relation between the self-gravity parameter $Q$ used in this paper and the Toomre parameter $Q_{2 \mathrm{D}}$ for razor-thin disks.

We first express the continuity equation in terms of horizontal velocity, density and potential perturbations. The vertical velocity perturbation is

$$
\delta v_{z}=\frac{\mathrm{i}}{\sigma}\left(\widetilde{W^{\prime}}+\epsilon V\right)
$$

where the linearized $y$ momentum equation was used (i.e. eliminating $\delta B_{y}^{\prime}$ between Eq. 19 and Eq. 20). Inserting this into the linearized continuity equation (Eq. 17), we obtain

$$
0=W^{\prime \prime}+(\ln \rho)^{\prime} W^{\prime}+\frac{\sigma^{2}}{c_{s}^{2}} W+\delta \Phi^{\prime \prime}+(\ln \rho)^{\prime} \delta \Phi^{\prime}+\sigma k_{x} \delta v_{x}+\epsilon D_{1} V .
$$

Next, we examine separately the cases of a vertical field with variable resistivity and that of a tilted field with uniform resistivity. (A similar procedure can be performed in the general case of a tilted field with variable resistivity.)

Vertical field with variable resistivity

First consider $\epsilon=0$ and $\eta=\eta(z)$ in the linearized equations. Denoting the $n^{\text {th }}$ vertical derivative as ${ }^{(n)}$, the equations of motion give

$$
\begin{aligned}
& \delta B_{x}^{(n)}=\frac{\mu_{0} \rho}{B_{z}} D_{n-1} U+\mathrm{i} k_{x} \delta B_{z}^{(n-1)}, \\
& \delta B_{y}^{(n)}=\frac{\mu_{0} \rho}{B_{z}} D_{n-1} V,
\end{aligned}
$$

for $n \geq 1$. Differentiating the divergence-free condition for the magnetic field gives

$$
\mathrm{i} k_{x} \delta B_{x}^{\prime}+\delta B_{z}^{\prime \prime}=0
$$

We insert the expression for $\delta B_{x}^{\prime}$ from Eq. C7 and the expression for $\delta B_{z}^{\prime \prime}$ from the $z$ component of the linearized induction equation (Eq. 23) to obtain

$$
-\sigma \delta B_{z}^{(n)}=k_{x} B_{z} \delta v_{x}^{(n)}+k_{x} \frac{\mu_{0} \rho}{B_{z}} \bar{D}_{n} U .
$$

Inserting the above expressions for $\delta B_{x}^{\prime \prime}, \delta B_{x}^{\prime}\left(\right.$ Eq. C7) and $\delta B_{z}^{\prime}$ (Eq. C10) into the right-hand-side of the $x$-induction equation (Eq. 21) gives

$$
\mathrm{i} \sigma \delta B_{x}=B_{z} \delta v_{x}^{\prime}+\frac{\mu_{0} \rho}{B_{z}} \bar{D}_{1} U .
$$


$(\bar{\sigma} \neq 0$ has been assumed to obtain this.) We differentiate this expression with respect to $z$ and eliminate the resulting $\delta B_{x}^{\prime}$ using Eq. C7, to obtain

$$
0=v_{A}^{2}\left(\delta v_{x}^{\prime \prime}-k_{x}^{2} \delta v_{x}\right)+\left(\bar{D}_{2}-k_{x}^{2} \bar{D}_{0}-\mathrm{i} \sigma D_{0}\right) U
$$

We follow a similar procedure as above to remove $\delta B_{y}$. We use Eq. C11 and Eq. C8 to eliminate $\delta B_{x}, \delta B_{y}^{\prime}$ and $\delta B_{y}^{\prime \prime}$ from the right-hand-side of the $y$-induction equation (Eq. 22),

$$
\mathrm{i} \bar{\sigma} \delta B_{y}=B_{z} \delta v_{y}^{\prime}+\frac{\mathrm{i} S}{\sigma}\left(B_{z} \delta v_{x}^{\prime}+\frac{\mu_{0} \rho}{B_{z}} \bar{D}_{1} U\right)+\frac{\mu_{0} \rho}{B_{z}} \bar{D}_{1} V .
$$

We differentiate this expression with respect to $z$, then eliminate $\delta B_{y}$ and $\delta B_{y}^{\prime}$ from the left-hand-side of the resulting expression using Eq. C13 and Eq. C8, respectively. We obtain

$$
0=v_{A}^{2}\left(\delta v_{y}^{\prime \prime}-\frac{\bar{\sigma}^{\prime}}{\bar{\sigma}} \delta v_{y}^{\prime}\right)+\frac{\mathrm{i} S v_{A}^{2}}{\sigma}\left(\delta v_{x}^{\prime \prime}-\frac{\bar{\sigma}^{\prime}}{\bar{\sigma}} \delta v_{x}^{\prime}\right)+\frac{\mathrm{i} S}{\sigma}\left(\bar{D}_{2}-\frac{\bar{\sigma}^{\prime}}{\bar{\sigma}} \bar{D}_{1}\right) U+\left(\bar{D}_{2}-\frac{\bar{\sigma}^{\prime}}{\bar{\sigma}} \bar{D}_{1}-\mathrm{i} \bar{\sigma} D_{0}\right) V .
$$

Eq. C12 and C14 constitutes the first two linearized equations to be solved.

\section{Tilted field with uniform resistivity}

Here we allow $\epsilon \neq 0$ but take $\eta$ to be constant. We first obtain expressions for $\delta B_{x}$ and $\delta B_{y}$. Differentiating the $x$ momentum equation and replacing the resulting $\delta B_{z}^{\prime}$ using the divergence-free condition and $\delta B_{y}^{\prime}$ using the $y$ momentum equation, we obtain an expression for $\delta B_{x}^{\prime \prime}$ which can be inserted into the $x$ induction equation. This gives

$$
\mathrm{i} \sigma \delta B_{x}=B_{z} \delta v_{x}^{\prime}+\frac{\eta \mu_{0} \rho}{B_{z}}\left(D_{1} U+\mathrm{i} \epsilon k_{x} D_{0} V\right) .
$$

We can insert this into the $y$ induction equation to obtain

$$
\mathrm{i} \bar{\sigma} \delta B_{y}=-B_{y} \Delta+B_{z} \delta v_{y}^{\prime}+\frac{\mathrm{i} S}{\sigma}\left[B_{z} \delta v_{x}^{\prime}+\frac{\eta \mu_{0} \rho}{B_{z}}\left(D_{1} U+\mathrm{i} \epsilon k_{x} D_{0} V\right)\right]+\frac{\eta \mu_{0} \rho}{B_{z}} D_{1} V
$$

where we have also used the derivative of the $y$ momentum equation to eliminate $\delta B_{y}^{\prime \prime}$. Recall $\Delta \equiv i k_{x} \delta v_{x}+\delta v_{z}^{\prime}$, so that

$$
\Delta=\mathrm{i} k_{x} \delta v_{x}+\frac{i}{\sigma}\left(\widetilde{W^{\prime \prime}}+\epsilon V^{\prime}\right)=-\left[\frac{\mathrm{i} \sigma W}{c_{s}^{2}}+\frac{\mathrm{i}(\ln \rho)^{\prime}}{\sigma}\left(\widetilde{W^{\prime}}+\epsilon V\right)\right]
$$

where the second equality results from the continuity equation.

Now consider

$$
\delta B_{x}^{\prime}-\mathrm{i} k_{x} \delta B_{z}=\frac{\mu_{0} \rho}{B_{z}} D_{0} U+\mathrm{i} \epsilon k_{x} \delta B_{y}=\frac{\sigma}{\bar{\sigma}} \delta B_{x}^{\prime}+\frac{\mathrm{i} k_{x}^{2} B_{z}}{\bar{\sigma}} \delta v_{x}
$$

where the first equality corresponds to the $x$ momentum equation and the second equality results from replacing $\delta B_{z}$ using the $z$ induction equation. We can now use the above expressions for $\delta B_{x}$ and $\delta B_{y}$ (Eq. C15 C16) to obtain

$$
\begin{aligned}
0= & v_{A}^{2}\left[k_{x}^{2}\left(1+\epsilon^{2}\right) \delta v_{x}-\frac{\epsilon k_{x} S}{\sigma} \delta v_{x}^{\prime}-\delta v_{x}^{\prime \prime}\right]+\mathrm{i} \epsilon k_{x} v_{A}^{2} \delta v_{y}^{\prime}+\frac{\epsilon^{2} k_{x} v_{A}^{2}}{\sigma}\left(\widetilde{W^{\prime \prime}}+\epsilon V^{\prime}\right) \\
& -\left[\eta\left(D_{2}+\frac{\epsilon k_{x} S}{\sigma} D_{1}\right)-\mathrm{i} \bar{\sigma} D_{0}\right] U-\frac{\mathrm{i} \epsilon^{2} k_{x}^{2} S}{\sigma} \eta D_{0} V .
\end{aligned}
$$

Similarly, we differentiate Eq. C16 and use the $y$ momentum equation to eliminate $\delta B_{y}^{\prime}$ to obtain

$$
\begin{aligned}
0= & v_{A}^{2} \delta v_{y}^{\prime \prime}+\frac{\mathrm{i} S}{\sigma} v_{A}^{2} \delta v_{x}^{\prime \prime}+\frac{\mathrm{i} S}{\sigma} \eta D_{2} U+\left\{\eta\left(D_{2}-\frac{\epsilon k_{x} S}{\sigma} D_{1}\right)+\mathrm{i}\left[\frac{\epsilon^{2} v_{A}^{2}(\ln \rho)^{\prime \prime}}{\sigma}-\bar{\sigma}\right] D_{0}\right\} V \\
& +\mathrm{i} \epsilon v_{A}^{2}\left\{\frac{\sigma}{c_{s}^{2}}\left[W^{\prime}-\left(\ln c_{s}^{2}\right)^{\prime} W\right]+\frac{1}{\sigma}\left[(\ln \rho)^{\prime}\left(\widetilde{W^{\prime \prime}}+\epsilon V^{\prime}\right)+(\ln \rho)^{\prime \prime} \widetilde{W^{\prime}}\right]\right\} .
\end{aligned}
$$

\section{REFERENCES}

Abramowitz, M., \& Stegun, I. A. 1965, Handbook of mathematical functions with formulas, graphs, and mathematical tables, ed.

Abramowitz, M. \& Stegun, I. A.

Aizenman, M., Smeyers, P., \& Weigert, A. 1977, A\&A, 58, 41

Armitage, P. J. 2011, ARA\&A, 49, 195

Armitage, P. J., Livio, M., \& Pringle, J. E. 2001, MNRAS, 324, 705 
Balbus, S. A., \& Hawley, J. F. 1991, ApJ, 376, 214

-. 1998, Reviews of Modern Physics, 70, 1

Balbus, S. A., \& Papaloizou, J. C. B. 1999, ApJ, 521, 650

Bonanno, A., \& Urpin, V. 2007, ApJ, 662, 851

Boss, A. P. 1997, Science, 276, 1836

-. 1998, ApJ, 503, 923

Chandrasekhar, S. 1961, Hydrodynamic and hydromagnetic stability, ed. Chandrasekhar, S.

Christodoulou, D. M. 1993, ApJ, 412, 696

Elmegreen, B. G. 1987, ApJ, 312, 626

Fan, Z., \& Lou, Y.-Q. 1997, MNRAS, 291, 91

Fleming, T., \& Stone, J. M. 2003, ApJ, 585, 908

Fromang, S. 2005, A\&A, 441, 1

Fromang, S., Balbus, S. A., \& De Villiers, J.-P. 2004a, ApJ, 616, 357

Fromang, S., Balbus, S. A., Terquem, C., \& De Villiers, J.-P. 2004b, ApJ, 616, 364

Fromang, S., de Villiers, J. P., \& Balbus, S. A. 2004c, Ap\&SS, 292, 439

Fromang, S., Terquem, C., Balbus, S. A., \& de Villiers, J.-P. 2004d, in Astronomical Society of the Pacific Conference Series, Vol. 321, Extrasolar Planets: Today and Tomorrow, ed. J. Beaulieu, A. Lecavelier Des Etangs, \& C. Terquem, 262

Gammie, C. F. 1996a, ApJ, 457, 355

-. 1996b, ApJ, 462, 725

-. 2001, ApJ, 553, 174

Gammie, C. F., \& Balbus, S. A. 1994, MNRAS, 270, 138

Goldreich, P., \& Lynden-Bell, D. 1965a, MNRAS, 130, 97

-. 1965b, MNRAS, 130, 125

Goodman, J. 2003, MNRAS, 339, 937

Goodman, J., \& Xu, G. 1994, ApJ, 432, 213

Gressel, O., Nelson, R. P., \& Turner, N. J. 2012, MNRAS, 422, 1140

Helled, R., et al. 2013, ArXiv e-prints

Inutsuka, S.-i., Machida, M. N., \& Matsumoto, T. 2010, ApJ, 718, L58

Jin, L. 1996, ApJ, 457, 798

Kim, J.-G., Kim, W.-T., Seo, Y. M., \& Hong, S. S. 2012, ApJ, 761, 131

Kim, W.-T., \& Ostriker, E. C. 2000, ApJ, 540, 372

-. 2001, ApJ, 559, 70

Kim, W.-T., Ostriker, E. C., \& Stone, J. M. 2003, ApJ, 599, 1157

Kojima, Y., Miyama, S. M., \& Kubotani, H. 1989, MNRAS, 238, 753

Landry, R., Dodson-Robinson, S. E., Turner, N. J., \& Abram, G. 2013, ApJ, 771, 80

Lin, M.-K. 2012, ApJ, 754, 21

Lizano, S., Galli, D., Cai, M. J., \& Adams, F. C. 2010, ApJ, 724, 1561

Lubow, S. H., \& Martin, R. G. 2012, ApJ, 749, L37

Lynden-Bell, D., \& Kalnajs, A. J. 1972, MNRAS, 157, 1

Mamatsashvili, G. R., \& Rice, W. K. M. 2010, MNRAS, 406, 2050

Martin, R. G., Lubow, S. H., Livio, M., \& Pringle, J. E. 2012a, MNRAS, 420, 3139

-. 2012b, MNRAS, 423, 2718

Menou, K., \& Quataert, E. 2001, ApJ, 552, 204

Nakamura, T. 1983, Progress of Theoretical Physics, 70, 747

Ogilvie, G. I. 1998, MNRAS, 297, 291

Papaloizou, J. C., \& Savonije, G. J. 1991, MNRAS, 248, 353

Papaloizou, J. C. B., \& Lin, D. N. C. 1989, ApJ, 344, 645

Pessah, M. E., \& Psaltis, D. 2005, ApJ, 628, 879

Reyes-Ruiz, M. 2001, ApJ, 547, 465

Sano, T., \& Miyama, S. M. 1999, ApJ, 515, 776

Terquem, C. E. J. M. L. J. 2008, ApJ, 689, 532

Toomre, A. 1964, ApJ, 139, 1217

Turner, N. J., Fromang, S., Gammie, C., Klahr, H., Lesur, G., Wardle, M., \& Bai, X.-N. 2014, ArXiv e-prints Vorobyov, E. I. 2013, A\&A, 552, A129

Yang, C.-C., Mac Low, M.-M., \& Menou, K. 2012, ApJ, 748, 79

Zhu, Z., Hartmann, L., \& Gammie, C. 2010a, ApJ, 713, 1143

Zhu, Z., Hartmann, L., Gammie, C. F., Book, L. G., Simon, J. B., \& Engelhard, E. 2010b, ApJ, 713, 1134 